\author{
Military Technical College
}

Kobry Elkobbah, Cairo,

Egypt.

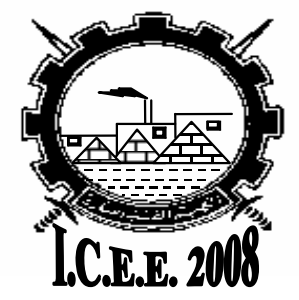

$4^{\text {th }}$ International Conference On

Chemical \& Environmental Engineering

27-29 May 2008

\title{
SELF-ASSEMBLED SYSTEMS: CHEMICAL ENGINEERING OF SURFACES FOR DEVICE FABRICATION
}

\author{
M. Abdel-Mottaleb
}

\begin{abstract}
:
The control of the lateral assembly and spatial arrangement of micro- and nanoobjects at interfaces is a prerequisite for potential applications in the field of nanoscience and technology. To create two-dimensional patterns, one can take advantage of active manipulation techniques, such as photolithography, electron beam lithography, and soft lithography. Self-assembly methods provide an alternative approach to build defined structures with dimensions on the nanometer scale. Selfassembly is a natural phenomenon that can be observed in many biological, chemical, and physical processes. Self-assembled monolayers (SAMs) are ordered molecular assemblies formed by the adsorption of an active surfactant on a solid surface. In this contribution, different approaches for building, characterizing and manipulating SAMs will be demonstrated. Scanning tunneling microscopy (STM) technique has been utilized in this study.
\end{abstract}

\section{KEYWORDS:}

STM, Self-assembly, Fabrication, Device, physisorption. 


\section{INTRODUCTION: STRUCTURAL ENCODING}

Self-organizing MOLECULAR systems are in the focus of nanotechnology research because of their potential use in "bottomup" approaches toward functional (supra)molecular devices. The design and synthesis of molecules that form selfassembled monolayers (SAMS) on substrates are fairly understood and developed. Scanning tunneling microscopy (STM) has emerged as one of the best tools to image, analyze, and control self-assembled molecular systems on substrates under various conditions (ambient, UHV, liquid-solid interface) with resolutions down to the (sub)molecular level.

\section{RESULTS AND DISCUSSION}

\section{Proof of Concept: Structural Encoding and Chemical Reactivity}

\section{A. 5((wperfluorohexyl)undecanyloxy)isophthalic acid (F6H11-ISA).}

Images of the lamellar structure of F6H11-ISA could be obtained by depositing a drop of a solution containing this compound on Highly Oriented Pyrolytic Graphite (HOPG). An STM image representative for the observed monolayer structure is shown in Figure 2a. The perfluorinated part of the alkyl chain can clearly be distinguished as a black band, due to a decreased tunneling current detected over the fluorinated methylene groups. This is in agreement with predictions that the fluorinated moiety will induce a change in contrast with respect to the hydrogenated moiety in the STM images when physisorbed on the basal plane of graphite. The observed bright spots correspond to the location of the isophthalic acid groups. The non-fluorinated part of the alkyl chain can be seen as a darker band with respect to the isophthalic acid groups. Bright and dark refers to the black/white contrast in the images. White corresponds to the highest and black to the lowest measured tunneling current in the image. The occurrence of a higher tunneling current above an aromatic moiety, as predicted by theoretical calculations, is a general finding, which has been observed for a large variety of organic adsorbates on graphite. Two different lamellar widths can clearly be distinguished. The wide lamellae consist of F6H11-ISA molecules while the narrow lamellae are built up by solvent molecules. Based on the observed Moiré pattern the parameters of the unit cell, indicated in the molecular model presented in Figure $2 b$, were determined to be $9.59 \pm 0.05 \AA, 52.3 \pm$ $2.6 \AA$ and $81 \pm 3^{\circ}$ respectively. The unit cell parameters $a, b$, and $\alpha$ obtained from molecular modelling are $9.60 \AA, 52.1 \AA$, and $81^{\circ}$ respectively. The experimental parameters are in good agreement with the molecular model. From the presented data it is clear that the fluorinated alkyl segments do not segregate into microdomains.

\section{B. Mixtures of F6H11-ISA with H16-ISA.}

Upon mixing F6H11-ISA with H16-ISA monolayers can again be observed. Both semifluorinated and non-fluorinated molecules can be recognized in the presented image by their characteristic STM contrast, arising from a modified tunneling current over specific areas of the monolayer. From the image it is clear that separate 
domains of non-fluorinated and semifluorinated molecules are not observed. Even though both species differ in length by one methylene unit the lamellae are built up by both fluorinated and non-fluorinated molecules which are interdigitating with each other, as can be seen in Figure 3. In the image presented in Figure 3 the nonfluorinated isophthalic acid derivative is the dominating species, although the mixture has a 1:1 concentration ratio. The same results were obtained for a 5:1 mixture of F6H11-ISA and H16-ISA respectively (not shown). There is no clear dependence of the composition of the two-dimensional co-crystal on the relative concentration of the components in solution. This is probably due to the preferential adsorption of the $\mathrm{CH}_{2}$ groups on graphite compared to the $\mathrm{CF}_{2}$ groups. Moreover, the hydrocarbon chain has a stronger interaction with the graphite surface since it has an all-trans conformation while the fluorocarbon chain has a helical conformation. Fluorinated molecules are incorporated randomly in the lamellae of the non-fluorinated isophthalic acid derivative. The semifluorinated alkyl chains are fully interdigitated with the non-fluorinated alkyl chains. This was observed for all the STM data obtained for the mixtures discussed in this section. Separate domains of either compound were never observed during the different sessions using different tips. The perfluorinated part of the alkyl chains can again be observed with dark contrast at the end of the molecules. This allows us to point out the exact location of each semifluorinated molecule that has been coadsorbed in monolayers of H16-ISA. A fluorinated molecule incorporated in a H16-ISA lamella is indicated by an arrow in Figure 3. The fluorine subsituted methylene groups can thus serve as a nonperturbative probe for the analysis of the structure of two-dimensional crystalline systems observed with STM.

The observation of miscibility of the protonated and fluorinated isophthalic acid is corroborated by the crystal structures. The ISA's $\mathrm{C}_{12}-\mathrm{C}_{16}$ form an isostructural series in which the lattice parameters change in a regular fashion adding an increment for each $\left(\mathrm{CH}_{2}-\mathrm{CH}_{2}\right)$ unit added. F6H11-ISA also crystallizes in this form. It should be noted that here the fluorinated segment has protonated next neighbors. Apparently the length of the $\mathrm{F} 6 \mathrm{H} 11$ segment is not sufficient to cause segregation.

\section{Mixtures of F6H11-ISA with H14-ISA.}

The only type of segregation observed is most likely caused by a significant difference in chain length of the molecules. This segregation was observed when a mixture of F6H11-ISA and H14-ISA in 1-octanol was investigated with STM. While in section $B$ the components in the mixture differ in length by only one methylene unit, there is now a difference of three methylene units. One of the obtained images is shown in Figure 4. Two types of lamella are visible. The wide lamellae (green arrow) are built up by F6H11-ISA molecules, as can be concluded from the typical features in the STM contrast that are characteristic to this molecule. The narrow lamella to the right of the image consists of H14-ISA molecules (red arrow). This is backed up through the analysis of the image. The width of the wider lamella is $35.42 \AA$, the width of the narrow lamella is $31.88 \AA$. This is in good agreement with values obtained from molecular modeling. In both types of lamellae the molecules are fully interdigitated. 
In contrast to the observations made for the mixture discussed in section $B$, in this case the dominating species in the monolayer are the fluorinated molecules. A small number of non-fluorinated molecules coadsorbed onto the surface in separate lamellae. For this mixture it was thus possible to observe lamellar segregation. An example of an area with domain boundaries is shown in Figure 4B. The lower resolution of this image in comparison to Figure 4A is caused by the mobility of the molecules at the domain boundaries. At this boundary, the domains show a slight angle $\left(4^{\circ}\right.$ at the domain boundary in the top-left of the image and $7^{\circ}$ at the domain boundary in the bottom-right of the image) with respect to each other. From this image one can clearly distinguish an isolated H14-ISA lamella incorporated in the F6H11-ISA domain in the center of the image. Domains consisting of only H14-ISA lamellae were never observed. From these data, which was observed during several sessions using different tips, we conclude that H14-ISA and F6H11-ISA do not segregate in to separate domains, but that H14-ISA forms separate lamellae in F6H11-ISA domains.

\section{F8H10-ISA and F12H11-ISA.}

Submolecular investigation of the two-dimensional organization of $5-((\omega-$ perfluorooctanyloxy)decanyloxy)isophthalic acid (F8H10-ISA) and 5- $((\omega-$ perfluorododecanyloxy)undecanyloxy)isophthalic acid (F12H11-ISA) has been carried out by means of STM at the liquid-solid interface. The molecular structures of the compounds under investigation are depicted in Figure 5.

Figure 6a shows an STM image of a physisorbed monolayer consisting of F8H10-ISA molecules adsorbed from a solution in 1-octanol onto the basal plane of graphite. The molecules are arranged in a bi-layer type structure with the lamellar width $\left(\Delta \mathrm{L}_{1}\right)$ equal to $43.3 \pm 3.8 \AA$. The observed bright spots (white circles) correspond to the location of the isophthalic acid groups. The occurrence of a higher tunneling current above an aromatic moiety, as predicted by theoretical calculations, is a general finding, which has been observed for a large variety of organic adsorbates on graphite. The difference in the apparent contrast (brightness) between the nonfluorinated alkyl chains at the left and right side of a row of isophthalic acid groups is a scanning artifact. The non-fluorinated alkyl chains form an angle of $45 \pm 4^{\circ}$ with respect to the lamella axis, which does not allow for interdigitation of the chains, leading to phase separation on the nano-scale (rows of isophthalic acid groups - nonfluorinated segments - fluorinated segments) (see Scheme 1a). A model for the observed packing pattern is presented in Figure 6b. Nanophase separation in this kind of systems is not trivial, in the sense that all 5-alkoxy-isophthalic acid derivatives investigated so far show full interdigitation of the alkylated part.

In time, during the imaging session, the monolayer structure changes into an interdigitated lamellar one. Figure 7a shows an STM image of such a monolayer. The specific contrast of F8H10-ISA can be clearly observed. The molecules now appear to be interdigitating. Thus there is a dynamic change in the monolayer packing pattern, from the nano-segregated patterns (Figure $7 \mathrm{a}$ ) to the interdigitating one 
(Figure 7a). The interdigitating pattern (Scheme 1b) was observed to be stable over extended periods; monolayers with nano-segregated pattern were never observed again in the same session. In Figure $7 \mathrm{a}$, two different lamellar widths can clearly be distinguished. The wide lamellae $\left(\Delta \mathrm{L}_{1}=34.2 \pm 1.2 \AA\right)$ consist of F8H10-ISA molecules while the narrow lamellae $\left(\Delta \mathrm{L}_{2}=16.3 \pm 0.5 \AA\right)$ are built up by solvent molecules. Interestingly, in all cases where the interdigitation of the alkyl chains was observed, the solvent molecules are coadsorbed. This suggests that, as a result of solvent co-adsorption, the hydrogen bonding involving the isophthalic acid groups overcomes the repulsive interactions between perfluorinated and non-fluorinated alkyl segments, leading to an interdigitated pattern. Figure $7 \mathrm{~b}$ shows a model for the packing pattern observed in Figure $7 a$.

Co-deposition of 1-alcohol molecules was observed for a substantial number of 5alkoxy-isophthalic acid derivatives. In a number of cases, we observed that the codeposition process leads to thermodynamically more stable 2D patterns. Often the first images obtained in a session do not show co-deposition of solvent molecules while later on, more co-deposited 1-alcohol solvent lamellae are formed. In a number of cases, it was even possible to follow this co-deposition process in real-time.

Based on the STM results obtained for F8H10-ISA molecules, one would expect that increasing the length of the perfluorinated segment results in favoring nanosegregation within the monolayer. Motivated by this idea, F12H11-ISA was investigated at the liquid-solid interface. It is clear that the expected packing pattern was not achieved as shown in a typical large scale STM image of a F12H11-ISA monolayer physisorbed at the liquid-solid interface (Figure 8a). The molecules appear to be fully interdigitating. Interestingly, the interdigitated pattern differs from the one of F8H10-ISA. The grouping of the molecules into units (Scheme 1c) consisting of eight molecules is evident. The perfluorinated segments appear with a dark contrast. In this particular image, the isophthalic acid groups are not as well resolved as in the higher resolution image in Figure $8 \mathrm{~b}$ where the bright spots correspond to the location of the isophthalic acid groups. The intermolecular distance -distance between equivalent isophthalic acid groups- within a unit was found to be $10.2 \pm 0.7 \AA$, which is slightly bigger than that typically found for isophthalic acid derivatives.19 This might be due to the longer perfluorinated segment, which will have more influence on the packing than in case of F8H10-ISA. A molecular model is provided in Figure 8c. As indicated earlier each unit consists of eight fully interdigitating molecules.

During the different sessions, solvent-free domains were never observed. Although it is not possible to rule out that they are formed, this suggests that they are not stable and easily converted to an adlayer structure with solvent co-deposition and interdigitation of the alkyl chains. Experiments in 1-phenyloctane, which is not known to codeposit, did not lead to the formation of stable monolayers. 
The STM image shown in Figure $8 d$ is of lower resolution with respect to the alkyl chains than that in Figure $8 \mathrm{a}$ and $8 \mathrm{~b}$, whereas the isophthalic acid groups and solvent lamellae are well-resolved. This is due to the mobility of the alkyl chains.

Apparently, increasing the length of the perfluorinated chain is the reason for the grouping of F12H11-ISA molecules into units. The incompatibility induced by the presence of the F12 segments should be strong enough to cause nano-segregation, but due to the long non-fluorinated segment $(\mathrm{H} 11)$ plus the hydrogen-bonding network, nano-segregation did not occur and interdigitated patterns (with solvent codeposition) were observed. However, the incompatibility induced by the F12 chains is expressed at two levels. First, the molecules group into units, until the lattice cannot tolerate the incompatibility induced by the F12 groups. It should be stressed that at the interface between two units, the intermolecular interactions are optimized, resulting in close contacts between the perfluorinated-perfluorinated and the nonfluorinated - non-fluorinated parts of the adjacent molecules, Figure 8b. Second, within the units, the contact between the non-fluorinated and fluorinated parts leads to less favorable enthalpic interactions, resulting in an increased mobility of the alkyl chains (Figure 8d). Thus, the increased mobility is a result of, and a clear demonstration of incompatibility between the perfluorinated segments and the nonfluorinated ones. The grouping of molecules into units has been previously observed for alkylcyanobiphenyls ( $\mathrm{mCB}, \mathrm{m}=8,10,12)$. Incommensurate packing was used to explain the formation of units and the packing pattern of the mCB molecules at the liquid-solid interface was explained as a compromise between the molecule-molecule interactions and the molecule-substrate interactions. Incommensurate packing is also proposed here to explain the clustering of F12H11-ISA molecules into units of eight at the liquid-solid interface. The strong incompatibility of perfluorinated and nonfluorinated segments, and the weak perfluorinated segments-substrate interactions tend to induce nano-segregation in the monolayer. At the same time, the interaction of non-fluorinated segments with the graphite surface in addition to the hydrogenbonding network formed between the isophthalic acid groups and solvent molecules are tending to induce interdigitation. Hence, the observed packing pattern of $\mathrm{F} 12 \mathrm{H} 11-$ ISA molecules at the liquid-solid interface is a subtle compromise between these opposing interactions within the monolayer.

\section{E. Chemical Reactivity.}

[2+2] Photodimerization reactions of cinnamic acid derivatives have been investigated extensively in three-dimensions (3D). The classic studies by Schmidt et al. have demonstrated that the packing of molecules in the crystals "strictly" controls such reactions. Some of these cinnamic acids dimerize upon irradiation with UV-light, while in solution trans-cis isomerization occurs but no dimerization (scheme 2). Based on extensive crystallographic and photochemical studies on cinnamic acids Schmidt et al. drew the following conclusions: (a) the nature of the crystal structure determines whether or not the reaction can occur and also determines the molecular structures of the products, if any; (b) the reaction involves the combination between nearest neighbor molecules in a stack, and occurs with a minimum of atomic and molecular movement. A reaction that behaves in this way is said to be 
topochemically controlled. These postulates imply that the reactive centers must be properly placed in the crystal for the reaction to occur, and that the topochemical processes are constrained by lattice forces which are assumed to maintain electronically excited molecules in structures close to the ones they adopted in their ground state. Cinnamic acid and its derivatives were observed to crystallize in three polymorphic forms, and show photochemical behavior which is determined by this structure type. The three structural types, namely $\alpha, \beta$, and $\gamma$, differ in the distance between equivalent points of neighboring molecules which is $\sim 4.2 \AA$ (centric double bond distance), 3.8-4.2 $\AA$, and 4.8-5.2 $\AA$, respectively.

These topochemical postulates are landmarks in organic solid state photochemistry and are used as rules, as they are able to provide an understanding of a large number of [2+2] photodimerization reactions of widely varying structures. However several examples do exist where the systems under investigation deviate significantly from the topochemical postulates suggested by Schmidt. In some cases the distance between the interacting double bonds is larger than the proposed upper limit for interaction (4.2 A) or the double bonds are not exactly parallel and yet the molecules photodimerize. Two approaches have been used to explain the deviations from the topochemical postulates, namely "reaction cavity" and "dynamic preformation".

STM was used to investigate the two-dimensional (2D) organization and photodimerization of cinnamic acid derivatives at the graphite/liquid interface. The photoreactivity of a symmetrically and an asymmetrically substituted cinnamic acid derivative was investigated. The difference in substitution is reflected in the $2 \mathrm{D}$ ordering and consequently also in the photoreactivity.

When a drop of the solution of C18CinnC18 in 1-octanol is applied to a freshly cleaved graphite surface, a physisorbed monolayer is spontaneously formed at the graphite/1-octanol interface. Figure 9a shows an image of such a monolayer observed with STM. The image is submolecularly resolved, which enables us to identify the cinnamate groups as well as the aliphatic chains. The brighter regions (higher tunneling current) in the STM image originate from the cinnamate moieties (indicated by a red arrow). The lamella is defined by two black troughs, which are characteristic for terminal methyl groups (indicated by the two yellow arrows). From the STM image the V-shape conformation of the molecule is clearly evident. To guide the eye, one C18CinnC18 molecule has been schematically drawn in the STM image. Based upon the contrast of the cinnamate groups in this image it is obvious that the phenyl rings are aligned in a row. So all molecules are equivalent along the lamella axis. A molecular model for the observed packing is shown in Figure $9 \mathrm{~b}$. The distance between two neighboring molecules within a lamella (a) is $6.3 \pm 0.5 \AA$, the lamella width $(\Delta \mathrm{L})$ is $54 \pm 3 \AA$, and the alkyl chains form an angle of $118 \pm 4^{\circ}$ with respect to the lamella axis as shown. These molecules do not form a true $2 \mathrm{D}$ crystal lattice, but rather parallel one-dimensional (1D) rows. The contrast of the cinnamate groups in some images such as in Figure 9c, indicates that C18CinnC18 can also arrange in a different way within the lamella. The STM image is of high resolution, individual aryl groups can clearly be discerned. The molecules in Figure 9a are packed in a way that the aryl groups in a given lamella are all lying along the same 
line (Type 1). On the other hand, close inspection of the STM image in Figure 9c reveals that the aryl groups in any given lamella are adopting a zigzag order (Type 2). Two molecules have been schematically drawn in the STM image. A molecular model representing the ordering of the molecules observed in this STM image is given in Figure 9d. The packing parameters of Type 2 do not differ from those of Type 1 within experimental error. The Type 2 orientation is not abundant but was imaged several times in different sessions.

In order to induce in-situ photodimerization of C18CinnC18, a UV-lamp (CAMAG universal lamp, wavelength $300 \mathrm{~nm}$ ) was used. Irradiation was carried out for different time intervals, which varied between $5 \mathrm{~min}$. and 5 days. Images observed after illumination for more than 10 minutes showed a new packing pattern (Figure 3a). To each bright structure (core, indicated by red arrows) four alkyl chains (indicated by the yellow lines) are connected. Based upon the STM contrast, it can be concluded that the alkyl chains adopt two different orientations. The plane formed by the carbon backbone of alkyl chains on one side of the core lies perpendicular to the surface, while the carbon backbone of the alkyl chains on the other side of the core lies parallel to the surface. At the left-bottom side of the image in Figure 10a, a change in the packing pattern of the adlayer can be observed. Two molecules are lying next to each other (the two molecules are pointed out with yellow arrows). Due to the change in the packing of the molecules in this lamella, there is a corresponding change in the neighboring lamella. The longer the irradiation time, the more of this new adlayer structure is observed. In order to understand the nature of the change in the packing pattern upon irradiation, solid crystals of $\mathbf{C 1 8 C i n n C 1 8}$ were irradiated at $300 \mathrm{~nm}$ up to 5 days and then dissolved in 1-octanol. Upon application of this solution to the graphite surface, the same features as observed in the STM image in Figure 10a are observed. Mass spectrometry showed the presence of dimers. These data strongly suggest that the new packing pattern is that of dimer adlayers. Upon ex-situ irradiation of highly diluted solutions $\left(\leq 10^{-6} \mathrm{M}\right)$ of $\mathbf{C 1 8 C i n n C 1 8}$, no evidence of dimer formation was detected neither by STM nor by other techniques (mass spectrometry). On the other hand ex-situ irradiation of more concentrated solutions ( $\geq$ $\left.10^{-5} \mathrm{M}\right)$ resulted in dimer formation. This was attributed to the formation of microcrystals. This suggests that upon in-situ irradiation of low concentrated solutions, the photodimerization of $\mathbf{C 1 8 C i n n C 1 8}$ occurs at the liquid/graphite interface.

There are two possible dimers of trans-C18CinnC18, namely $\alpha$-truxillic or $\beta$-truxinic derivatives (Scheme 2). The location of the bright structures suggests that they correspond to the core of the dimer molecule, which consists of the two phenyl rings and a cyclobutane ring. For an a-truxillic derivative, the core of the dimer is expected to appear in the STM image as two distinctive bright circles lying on opposite sides of a dark line going through the middle of the whole lamella. On the other hand, the $\beta$ truxinic derivative core is expected to appear as a U-shaped bright structure. In the STM image in Figure 10a, the bright structures in the center of a lamella appear to be U-shaped. Comparing this contrast with the structure of the two possible dimers (Scheme 2) suggests that the $\beta$-truxinic derivative is formed. A molecular model for 
the dimer molecule is proposed in Figure 10b. The packing parameters are $a=24.0$ $\pm 0.5 \AA, \Delta \mathrm{L}=61 \pm 4 \AA$.

Since the STM images suggest the formation of the $\beta$-truxinic derivative it must be trans-C18CinnC18 domains of Type 1 orientation which leads to this derivative. Type 2 should produce the $\alpha$-truxillic derivative, for which no evidence was found.

In addition to the experiments outlined above, the photodimerization of C18CinnC18 at the graphite/liquid interface has been visualized at an increased scan rate using a fast scanning STM (2 frames per second) which allows for simultaneous recording on a videotape. A detailed description of the fast scanning STM system is given elsewhere. In the experiments involving the modified STM, the samples were irradiated during scanning. A liquid light guide directs the light from a Xenon lamp, which is passed through heat filters and through a band pass filter [UG5 Schott filter, 250-400 nm] to the graphite substrate. During imaging, care was taken that the same region of the monolayer was imaged during the recording time. The packing pattern of the adlayer of the dimer molecules is very distinctive from that of the monomer molecule adlayers, which allows the clear differentiation between the two adlayers. In a first sequence of images (supporting information: movie1) obtained at a monomer/dimer domain boundary, the dimer molecules appear in a stepwise process. First, those monomer $\mathbf{C} 18 \mathrm{C}$ innC18 molecules closest to the domain boundary shared with the newly formed dimer domain, loose resolution due to enhanced mobility. This is followed by the appearance of dimer molecules at the same place that was previously occupied by the monomer molecules. In addition, photodimerization was observed in the bulk of the domain. In this case the dimer molecules were expelled from the adlayer, as the monomer adlayer cannot optimize the packing of the dimer molecules (supporting information: movie2).

In order to understand the effect of the 2D structure upon the photodimerization, C18CinnC10 was investigated, as it was expected to bias Type 1 over Type 2 packing due to its asymmetric structure. Figure 11a shows a STM image of the monolayer of C18CinnC10. The monolayer structure is different from the one obtained for C18CinnC18 (Figure 9). The decyl chains are clearly identified (indicated by yellow arrows) as being the shorter alkyl chains. They adopt a $90^{\circ}$ angle with respect to the lamella axis as opposed to $40^{\circ}$ for the octadecyl chain. Within a lamella, the $\mathbf{C} 18$ CinnC10 molecules have the decyl chains lying at the same side of the cinnamate group. The decyl chains are interdigitating while octadecyl chains are not. Figure $4 \mathrm{~b}$ shows the proposed molecular model of the observed monolayer: $\mathrm{a}=9.0 \pm 0.2 \AA, \Delta \mathrm{L}=49.9 \pm 0.8 \AA$. In contrast to C18CinnC18, C18CinnC10 monolayers did not show any change upon irradiation. In addition irradiation of concentrated solutions or solid crystals did not give any trace of dimer molecules.

According to the topochemical postulates and based upon the distances measured for both C18CinnC18 and C18CinnC10 monomer monolayers, neither of the two 
compounds was expected to photodimerize. Nevertheless, evidence provided by the STM experiments and the concentration dependent measurements strongly indicates that the photodimerization of $\mathbf{C 1 8 C i n n C 1 8}$ does take place within the monolayer. Two approaches were formulated to explain the reactivity of systems in $3 D$ that according to the topochemical postulates should not react. Cohen formulated "the role of the reaction cavity", according to which the topochemical postulates can be refined to "reactions proceeding under lattice control do so with minimal change or distortion of the surface of the reaction cavity". Gavezzotti has investigated the role of molecular environment in the crystal towards reactivity as well, and has generalized that a prerequisite for crystal reactivity is the availability of free space around the reaction site. In the second approach, Craig et al. pointed out the role of "dynamic preformation" which might influence product formation.Dynamical preformation can be summarized as follows: short-term lattice instability can have the effect of driving one molecule close to a neighbor so as to cause a photochemical reaction. This also can include rotation of the molecule into a more favorable orientation to allow the reaction to occur. Packing fluctuations are believed to be responsible for the photodimerization for a non-ideal monomer packing (distance $>4.2 \AA$, non-parallel double bonds).

Some of the STM images, which were acquired after 5 minutes of irradiation at 300 $\mathrm{nm}$, displayed a different packing pattern (Figure 12a) than those observed after longer irradiation period. The image allows us to identify the alkyl chains and the cinnamate groups in the lamellae. There is a lot of distortion in the image and in certain parts of the image, the distortion does not always allow the identification of individual cinnamate groups. Such a distortion is usually associated with a loose packing of the molecules (packing fluctuations), which leads to an increased mobility of the molecules or parts there of. Another indication of the high mobility of the molecules, is the variation in the lamella width. More importantly the images show that the conformation of the molecules is no longer V-shaped, instead the molecules appear to have adopted a linear conformation. The distance between adjacent molecules is found to be $3.9 \pm 0.2 \AA$, thus the aryl moiety must be tilted out of plane in order to allow this packing pattern. This explains the loss of resolution in the image, especially for the cinnamate groups, as the tilting of the molecules out of plane reduces the packing efficiency and hence increases the mobility of the molecules in the monolayer. ${ }^{8}$ This packing pattern also increases the overlap of the $\pi$-orbitals of the neighboring monomers, which is expected to facilitate dimerization. These observations emphasize the importance of packing fluctuations, where neighboring molecules have space to approach each other. Also, the loss of resolution in case of the fast scanning STM experiment followed by the appearance of the dimer molecules further points out the importance of packing fluctuations. However, a number of molecules are found to be interdigitating (Figure 12b). In this case the distance between successive molecules in the same lamella is found to be $8.5 \pm 0.4 \AA$, hence the aryl moieties can remain parallel to the graphite plane. In Figure 12 two molecules are schematically indicated. 
In case of C18CinnC10, the photostability can be explained as being due to interdigitation, leading to an increased intermolecular distance and hindering packing fluctuations.

\section{Template for Nanostructure Fabrication.}

Two-dimensional patterns of bipyridine derivatives: Urea groups are a robust motif for the formation of extended ribbons of hydrogen-bonded molecules. Compounds containing two urea groups separated by a spacer are particularly interesting, since they can form up to eight hydrogen bonds with neighboring molecules. From previous studies, it is clear that the intermolecular distance is often dictated by hydrogen bonding between the urea functionalities. On the basis of these previous findings, it was anticipated that the aromatic moieties would not lie flat in relation to the substrate, but rather would be tilted due to the limited space available. On the one hand, such an orientation could promote metal ion complexation at the liquid/solid interface, but on the other hand, the dominance of the hydrogen bonds might complicate the metal ion complexation. Motivated by these expectations and uncertainties, the 2D packing pattern of 1 (chemical structure: Scheme 3 ) at the liquid/solid interface was investigated. Upon applying a drop of a saturated solution of 1 in 1-phenyloctane or 1-octanol to the surface of graphite (HOPG), a well-ordered monolayer is spontaneously formed at the liquid/solid interface (Figure 13). The image is submolecularly resolved, which enables the identification of the different functionalities within the molecules. The molecules are ordered in rows, and the width of such a row (lamella) is indicated by $\Delta \mathrm{L}_{1}$. The bipyridine moieties appear as bright bands (black arrow) in the middle of the lamellae. They form an angle of $70 \pm 2 \circ$ with respect to the lamella axis. The urea groups appear as two bright lines (small arrows) on either side of the bipyridine moiety. The alkyl chains are fully extended and form an angle of $81 \pm 1 \circ$ with respect to the lamella axis. There is no indication of interdigitation. The intermolecular distance between two successive molecules within a lamella was found to be $4.6 \pm 0.3 \AA$, which is in good agreement with the intermolecular distances found in other urea-containing systems and is indicative of the formation of hydrogen bonds between the urea groups. Based on the packing parameters, a proposed model for the observed packing is depicted in Figure 13B. In this model, as expected, the intermolecular distance does not allow the bipyridine moieties to lie flat on the surface, and therefore a tilted arrangement is inferred. There is no information as to whether the nitrogen atoms of the pyridine moieties are oriented towards the graphite or the solution, nor on the eventual cisoid or transoid conformation adopted by the bipyridine. Urea-free bipyridine derivatives have also been investigated, the reason being that the absence of the urea groups puts less constraints on the intermolecular distance between adjacent bipyridine moieties, Scheme 3. Chemical structures of the bipyridine derivatives. and thereby might affect the metal ion complexation. Upon applying a drop of a solution of 2 in 1-phenyloctane to a graphite surface, a physisorbed monolayer is spontaneously formed at the liquid/solid interface. Figure 14 shows an image of such a monolayer, as observed by STM. The image is submolecularly resolved, which enables us to identify the two aromatic rings of the bipyridine moiety (indicated by the two big arrows) as well as the aliphatic chains. The lamella is defined by two black troughs, which are 
characteristic for terminal methyl groups (indicated by the two small arrows). The bipyridine moieties form an angle of $64 \pm 2{ }^{\circ}$ while the aliphatic chains form an angle of $49 \pm 2 \circ$, with respect to the lamella axis. For clarity, one molecular model of 2 has been superimposed on the STM image. Based on the contrast of the bipyridine moieties in the STM images it is obvious that all the molecules are equivalent along the lamella axis, and their chains appear to be fully extended. A molecular model of the observed packing is shown in Figure 14B. The distance between two neighboring molecules within a lamella measured along the lamella axis (a) is $6.9 \pm 0.3 \AA$, and the distance between equivalent points in abutting lamellae $\Delta \mathrm{L}_{1}$ is $51.0 \pm 1.6 \AA$. Note that the intermolecular distance is much larger (about a 50\% increase) compared to that in the case of 1 . The packing parameters acquired from the STM image indicate that the bipyridine moieties are adsorbed parallel to the graphite plane, and that there is no interdigitation of the aliphatic chains from neighboring lamellae. Again, the data do not provide information on the conformation of the bipyridine units. In Figure 14B, a cis conformation has been arbitrarily assumed, as this conformation will allow metal complexation.

In addition to the aforementioned highly uniform monolayers, some monolayers with packing irregularities were observed for 2 at the liquid/solid interface. Figure 15A and Figure 15B show lamellae of different widths. The small lamella shows the same packing pattern of 2 as observed in Figure 14A. In Figure 15A, two white arrows indicate what we believe to be the two pyridyl rings of the bipyridine moieties within a small lamella. The wider lamella $\left(\Delta \mathrm{L}_{2}=59 \pm 1.5 \AA\right)$ consists of rows of two molecules of 2 stacked along the lamella direction. Instead of just two lines of bright structures, three bright lines are visible. Two molecular structures are superimposed on the image for clarity. In this case, the bipyridine moieties are supposed to lie head-tohead with one alkyl chain per bipyridine moiety adsorbed on the graphite surface while the other alkyl chain is thought to be dangling in the supernatant solution (in this tentative model, only one alkyl chain is shown). At present, we have no explanation for this counterintuitive observation and cannot rule out the possibility that the bipyridine unit

may be partly tilted or even that part of the "visible" alkyl chain close to the bipyridine unit appears brighter because it is slightly lifted off the substrate. The width of the core in this case $(18.2 \pm 1.3 \AA)$ is consistent with this assumption.

To investigate the origin of the packing irregularities observed in the monolayers of 2 and the effect of molecular symmetry on the 2D ordering, monolayer formation by 3 , bearing only one alkyl chain (see Scheme 3), was investigated at the liquid/solid interface. Figure 16 shows an STM image of the monolayer of $\mathbf{3}$ observed at the liquid/solid interface. The lamellar structure of the monolayer is evident. The bipyridine moieties appear as bright bands in the middle of the lamella and the alkyl chains appear as somewhat darker than the bipyridine moieties. In this image, molecules adopt a head-to-tail packing as indicated by $\Delta \mathrm{L}_{1}(25.5 \pm 1.2 \AA)$ or a tail-totail packing as indicated by $\Delta \mathrm{L}_{2}(35.7 \pm 0.9 \AA)$. The intermolecular distance between neighboring molecules along a lamella axis is $6.5 \pm 0.4 \AA$. The alkyl chains are not interdigitated. 
In addition to the head-to-tail $\left(\Delta \mathrm{L}_{1}\right)$ and tail-to-tail $\left(\Delta \mathrm{L}_{2}\right)$ packings, double-core lamellae can be observed in the monolayer (Figure 17A: patterned arrows). In this case, the molecules are lying head-to-head. The core width is $16.7 \pm 1.1 \AA$, which is in good agreement with the width of two bipyridine moieties, whereas the width of a single core is $8.6 \pm 0.9 \AA$. Note that this core width (double core of 3 ) is identical (within experimental error) to the double-core width in the case of monolayers of 2 at the liquid/solid interface. The intermolecular distance between neighboring molecules along the lamella is $6.8 \pm 0.5 \AA$. Thus, there is no significant difference between lamellae with a head-to-head and those with a head-to-tail arrangement with respect to intermolecular distance. The alkyl chains are not interdigitated. In addition to the single-core and double-core lamellae observed for 3 at the liquid/solid interface, triple-core lamellae are also observed. Figure 17C shows a monolayer with such packing. A double-core lamella is indicated by the patterned arrows, while a triplecore lamella is indicated with black arrows. In the latter case, for the molecule adsorbed in the middle of the core, the alkyl chain will not be adsorbed on the surface. Thus, it is expected that the chain will be dangling in the supernatant solution. The width of the core $(28.7 \pm 1.5 \AA)$ confirms the proposed packing. The predominant packing, however, is the head-to-tail mode. These data confirm the packing irregularities observed for $\mathbf{2}$ and indicate that they are intrinsic properties of alkylated bipyridines.

Complexation with metal ions: Once the two-dimensional ordering of the bipyridine derivatives was established, we proceeded to investigate their complexation with metal ions at the liquid/solid interface. A drop of a concentrated solution of the chosen metal ion was added in situ to the preformed bipyridine monolayer. In the case of 1, in situ addition of complexed metal ions $\left(\mathrm{Pd}(\mathrm{OAc})_{2}, \mathrm{PdCl}_{2}, \mathrm{Cu}(\mathrm{OAc})_{2}\right.$, and $\mathrm{CuCl}_{2}$ ) in 1-phenyloctane solutions did not result in any observable change in either the contrast or the packing pattern and parameters of the monolayers of 1. Ex situ complexation of $\mathbf{1}$ did not result in the formation of a monolayer with different packing. Apparently, the close packing of the bipyridine moieties does not leave enough space for metal complexation to occur, and, most probably, the gain in free energy that would be achieved by metal complexation would not compensate for the loss of eight hydrogen bonds in the bipyridine-bis(urea) lamellae. Such a change in packing pattern would be necessary for the formation of the complex, and to allow for the accommodation of the metal atom within the monolayer. Another possibility might be the desorption of the complexed molecules from the surface.

After the successful imaging of monolayers of 2 at the 1-phenyloctane/graphite interface, a drop of a solution of $\mathrm{Pd}(\mathrm{OAc})_{2}$ in 1-phenyloctane was applied. Within a couple of minutes, a spontaneous change in the monolayer packing pattern could be observed. Figure 18A shows an STM image of such a monolayer of 2 after the addition of $\mathrm{Pd}(\mathrm{OAc})_{2}$. Two domains can be identified, domain $\mathrm{A}$ with a packing pattern similar to that previously observed for 2 (Figure 14A) and domain B with a new packing pattern, which is attributed to a bipyridine-metal complexed monolayer. In domain $B$, the core-to-core distance between abutting lamellae $\left(\Delta \mathrm{L}_{2}=35.1 \pm 1.3 \AA\right)$ is smaller than that previously observed for $2\left(\Delta \mathrm{L}_{1}=51.0 \pm 1.6 \AA\right)$. At the domain boundary, increased mobility of the molecules in some locations is evident (arrows). 
Figure 18B shows a large-scale image of the different domains observed after the addition of $\mathrm{Pd}(\mathrm{OAc})_{2}$. Domains $\mathrm{A}, \mathrm{B}, \mathrm{C}$, and $\mathrm{D}$ are assigned to the uncomplexed monolayer, while domain $\mathrm{E}$ corresponds to a complexed monolayer. It should be noted that the co-existence of complexed and uncomplexed domains is only observed immediately after the addition of the complexing agent $\mathrm{Pd}(\mathrm{OAc})_{2}$. In time, only monolayers with a packing pattern similar to that of domain B in Figure 18A (or domain $\mathrm{E}$ in Figure 18B) are observed. After the addition of the complexing agent, no packing irregularities were observed in the monolayer. Figure $18 \mathrm{C}$ shows an STM image of the monolayer with the new packing pattern. The image is submolecularly resolved, which again enables us to identify the aliphatic chains as well as the complexation sites. The bipyridine moieties cannot be discerned; instead, welldefined big bright structures appear (bright). The distance between two successive bright structures measured along the lamella axis (a) is $9.4 \pm 0.1 \AA$, which is significantly larger than the distance of $6.9 \pm 0.3 \AA$ between bipyridine moieties before the addition of $\mathrm{Pd}(\mathrm{OAc})_{2}$. A change in the packing pattern of the aliphatic chains occurs too, and the chains appear to be interdigitated. The data suggest that one bipyridine unit complexes to each metal complex. This can be explained as follows: the distance between the neighboring molecules increases to $9.4 \AA$ in order to accommodate the $\mathrm{Pd}(\mathrm{OAc})_{2}$ moieties, causing the aliphatic chains to interdigitate in order to reduce the free space in the monolayer, as is revealed by the large decrease in distance between adjacent rows of bipyridine units $\left(\Delta \mathrm{L}_{2}=35.1 \pm 1.3 \AA\right)$. It must be noted that the orientation of the aliphatic chains with respect to the lamellar axis changes from $49 \pm 2 \circ$ to $87 \pm 2 \circ$ after addition of $\mathrm{Pd}(\mathrm{OAc})_{2}$. To further clarify the packing pattern, two complexed molecules have been superimposed on the STM image. A molecular model of the observed monolayer packing is shown in Figure 18D.

Furthermore, in situ complexation of 2 with various complexed metal ions such as $\mathrm{PdCl}_{2}, \mathrm{Cu}(\mathrm{OAc})_{2}$, and $\mathrm{CuCl}_{2}$ was successfully accomplished. Figure 19A and Figure $19 \mathrm{~B}$ are STM images of $\left[\mathrm{Pd}(\mathrm{Cl})_{2}(\mathrm{C} 19 \mathrm{bipyC} 19)\right]$ monolayers. The STM images reveal a similar pattern as found for $\left[\mathrm{Pd}(\mathrm{OAc})_{2}(\mathrm{C} 19 \mathrm{bipyC} 19)\right]$ monolayers, with the same unit cell parameters. Figure 18C and Figure 18D show characteristic STM images of $\left[\mathrm{Cu}(\mathrm{OAc})_{2}(\mathrm{C} 19 \mathrm{bipyC} 19)\right]$ monolayers, while Figure 19E and Figure 19F show the corresponding images for $\left[\mathrm{Cu}(\mathrm{Cl})_{2}(\mathrm{C} 19\right.$ bipyC19)]. Again, the unit cell parameters are not significantly changed. This is quite remarkable given that the nature of the counterion is also different. This robustness in the complexation pattern formed is promising for future complexation experiments, allowing finetuning of the complexation properties by using different transition metals. In the case of the in situ addition of the metal complex, the new packing is thought to arise following desorption and readsorption of the molecules in close proximity to the surface.

To accurately compare the behavior of $\mathbf{3}$ with that of $\mathbf{2}$ at the liquid/solid interface, complexation of the former with $\mathrm{Pd}(\mathrm{OAc})_{2}$ was also investigated. A spontaneous change in the packing was observed, as depicted in Figure 20A. The molecules are packed in a lamellar-type structure, in which the alkyl chains are interdigitated; two arrows indicate a lamella. Figure 20B also shows an STM image of the complexed monolayer of 3. The intermolecular distance between neighboring molecules along the lamella is $9.2 \pm 0.5 \AA$. This value is identical (within experimental error) to that 
observed for the complexed monolayer of 2. Remarkably, after complexation, no different packings are observed; all domains show the same packing. Addition of the metal salts and consequent complexation leads to the formation of a unique 2D pattern.

To exploit the complexed monolayer as a template for building nanostructures, stability of the formed template in air would be a necessity. To assess this stability, a solution of $\mathbf{2}$ in 1-heptanol was applied to a graphite surface and left to dry under ambient conditions. Following this procedure, STM images similar to those obtained at the liquid/solid interface could be obtained. This does not prove that monolayer films were formed, although locally this is possible. More probably, the STM tip penetrates the deposited material and only the layer in contact with the graphite support is imaged. The observed monolayer was submolecularly resolved, and the packing parameters of the monolayer did not differ from those obtained at the liquid/solid interface. A drop of a $\mathrm{Pd}(\mathrm{OAc})_{2}$ solution in 1-heptanol was then applied on top of the dry layer, and again left to dry under ambient conditions for two days. It seems probable that the formation of the complexed layer involves desorption of the non-complexed molecules and readsorption of the complexed ones, driven by changes in the concentration gradient of complexed and non-complexed molecules in the supernatant solution. Figure 21 shows an image of the complexed monolayer acquired by STM. The image shows similar features to those obtained at the liquid/solid interface. It is also possible to induce decomplexation at the liquid/ solid interface and to restore the metal-free monolayer. This can be achieved, for example, by adding dibromomaleic acid in situ. Addition of dibromomaleic acid leads to a change in the packing pattern of the monolayer. The interdigitated packing pattern of the complexed monolayer is changed to the non-interdigitated one, characteristic of a non-complexed monolayer, indicating the in situ decomplexation of the monolayer by the dibromomaleic acid.

\section{CONCLUSION:}

It has been shown that chemical encoding of structure data is a very efficient and reliable method to control nanostructures on surfaces. Further, chemical reactions at the surface were controlled via variation in the structure without further chemical intervention.

Utilizing such control methods, a stable template for precise nanostructure construction was fabricated. The results reported here enable the fabrication of complex nanostructures possibly for device fabrication. 


\section{REFERENCES:}

[1] Steven De Feyter, André Gesquière, Mohamed M. Abdel-Mottaleb, Petrus C. M. Grim, Christian Meiners, Michel Sieffert, Suresh Valiyaveettil, Klaus Müllen and Frans C. De Schryver. Acc. Chem. Res. 2000, 33, 520-531, and references therein.

[2] Mohamed M. S. Abdel-Mottaleb, Steven De Feyter, André Gesquière, Michel Sieffert, Klaus Müllen, and Frans C. De Schryver. Nanoletters 2001, 1, 353-359, and references therein.

[3] Mohamed M. S. Abdel-Mottaleb, Steven De Feyter, Michel Sieffert, Markus Klapper, Klaus Müllen, and Frans C. De Schryver. Langmiur 2003, 19, 82568261, and references therein.

[4] Mohamed M. S. Abdel-Mottaleb, Elba Gomar-Nadal, Steven De Feyter, Magdalena Zdanowska, Jaume Veciana, Concepció Rovira, David B. Amabilino, and Frans C. De Schryver. Nanoletters 2003, 3, 1375-1378, and references therein.

[5] Mohamed M. S. Abdel-Mottaleb, Elba Gomar-Nadal, Mathieu Surin, Hiroshi UjiI,Wael Mamdouh, Jaume Veciana, Vincent Lemaur, Concepció Rovira, Jérôme Cornil, Roberto Lazzaroni, David B. Amabilino, Steven De Feyter, and Frans C. De Schryver. J. Mater. Chem. 2005, 15, 4601-4615, and references therein.

[6] Mohamed M. S. Abdel-Mottaleb, Günther Götz, P. Kilickiran, Peter Bäuerle and Elena Mena-Osteritz. Langmuir 2006 , 22, 1443-1448, and references therein.

[7] David B Amabilino, Steven De Feyter, Roberto Lazzaroni, Elba Gomar-Nadal, Jaume Veciana, Concepci'o Rovira, Mohamed M Abdel-Mottaleb, Wael Mamdouh, Patrizia lavicoli, Krystallia Psychogyiopoulou, Mathieu Linares, Andrea Minoia, Hong Xu and Josep Puigmart. J. Phys.: Condens. Matter 2007, and references therein. 
(a)

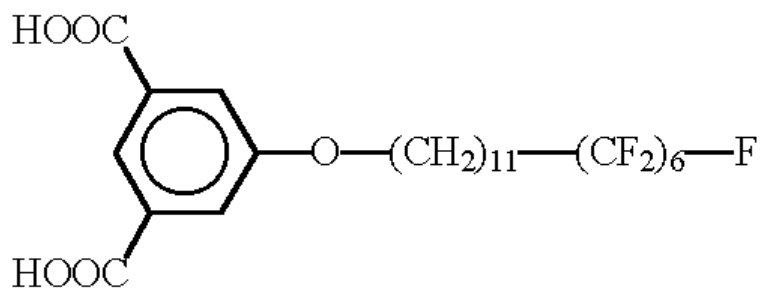

(b)

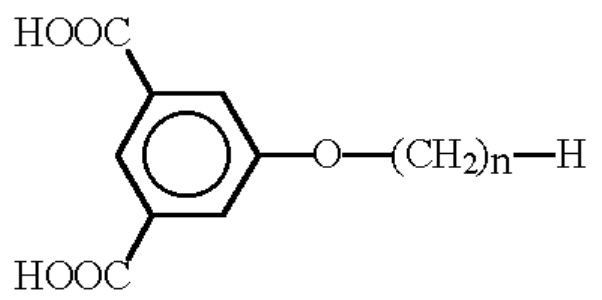

Figure 1. Chemical structure of (a) F6H11-ISA, and (b) Hn-ISA, $n=14,16$.

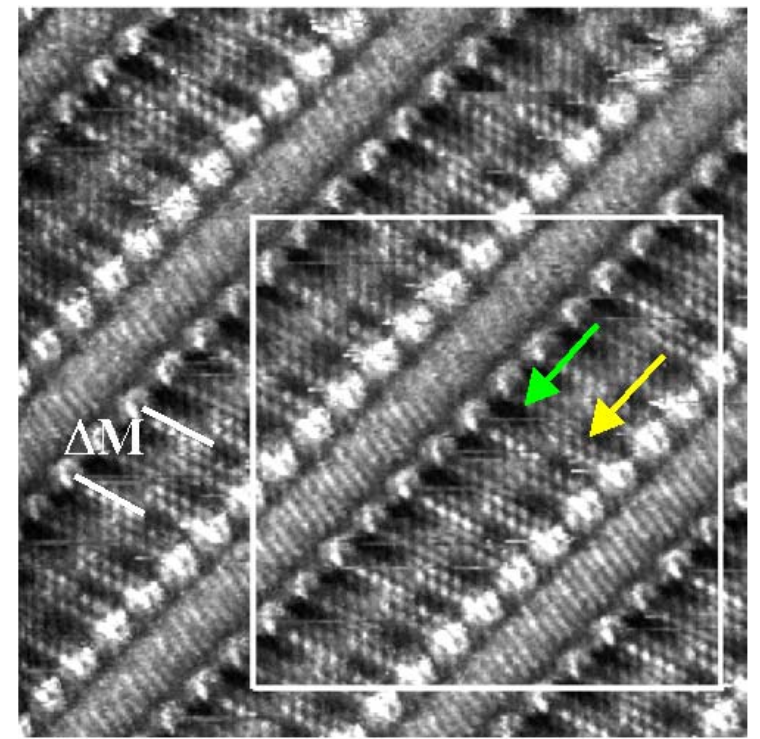

(a)

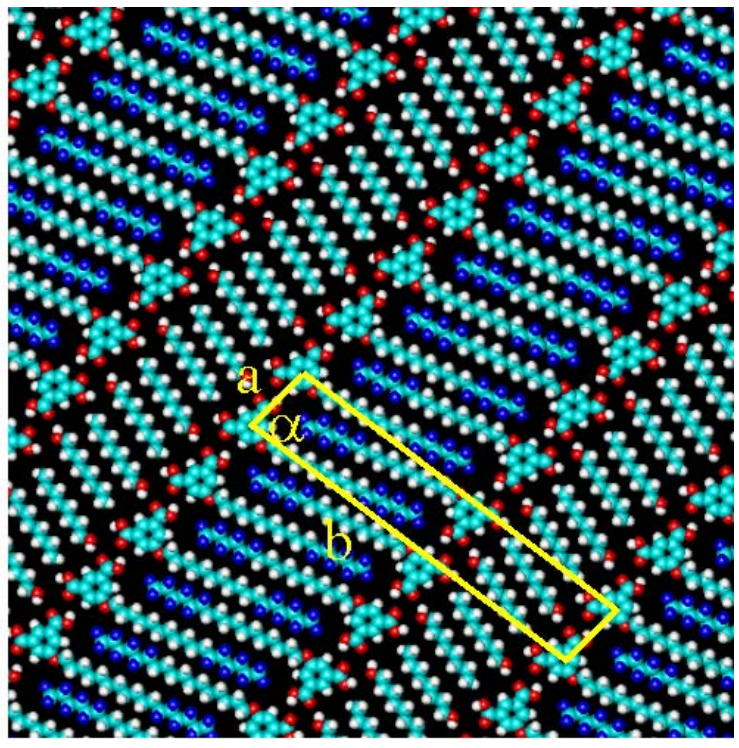

(b)

Figure 2. (a) STM image of a monolayer of F6H11-ISA adsorbed from a solution in 1octanol on to a freshly cleaved graphite surface. This submolecularly resolved image allows us to distinguish the different functional groups within a single molecule based on the characteristic contrast these groups exhibit in STM images. The green arrow points out the fluorinated part of the alkyl chain, while the non-fluorinated part is pointed out by the yellow arrow. Lamellae of F6H11-ISA are interspersed with lamellae built up by 1-octanol molecules. The Moiré period is indicated by $\Delta \mathrm{M}$, and contains 4 alkylchains and 9 main graphite axis. The image size is $13.1 \times 13.1 \mathrm{~nm} 2$. Iset $=1.0 \mathrm{nA}$, and Vbias $=696 \mathrm{mV}$. (b) Molecular model representing the area indicated in the STM image. Unit cell parameters $a$, b, and $\alpha$ are $9.59 \pm 0.30 \AA, 52.1$ $\pm 0.7 \AA$, and $81 \pm 2^{\circ}$ respectively. 


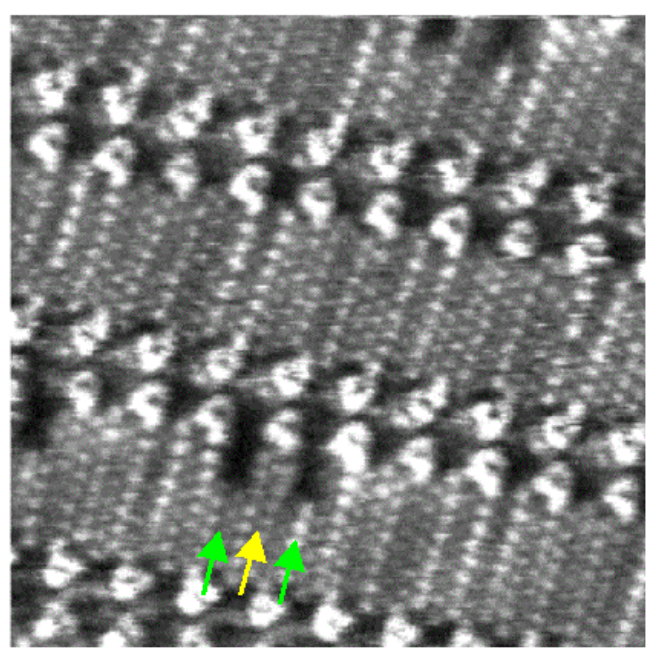

Figure 3. Monolayer of a mixture of F6H11-ISA with H16-ISA adsorbed from 1-octanol on to the graphite surface. The image reveals a densely packed structure in which the molecules are all interdigitating, regardless of being perfluorinated or non-fluorinated molecules. Two fluorinated molecules are indicated by the green arrows, a H16-ISA molecule is indicated by the yellow arrow. The image size is $9.7 \times 9.7 \mathrm{~nm}^{2}$. $\mathrm{I}_{\text {set }}=1.3 \mathrm{nA}$, and $\mathrm{V}_{\text {bias }}=1.35 \mathrm{~V}$.

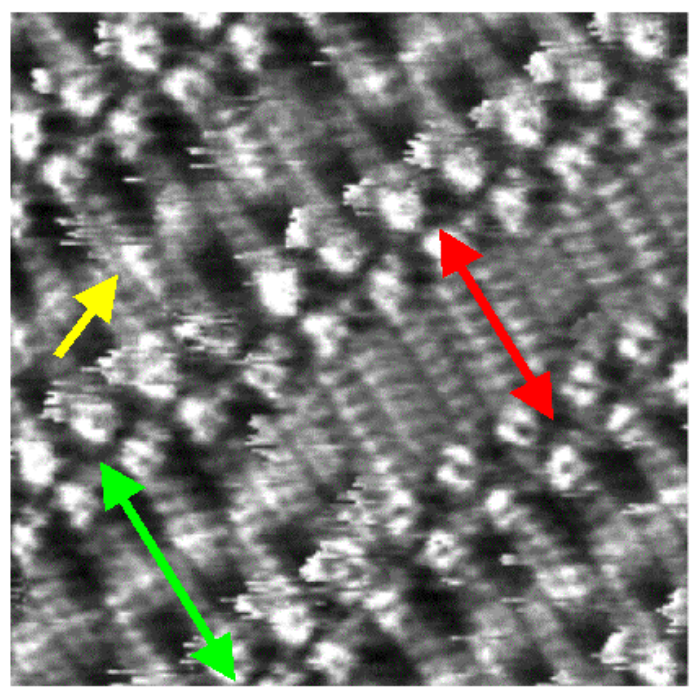

(a)

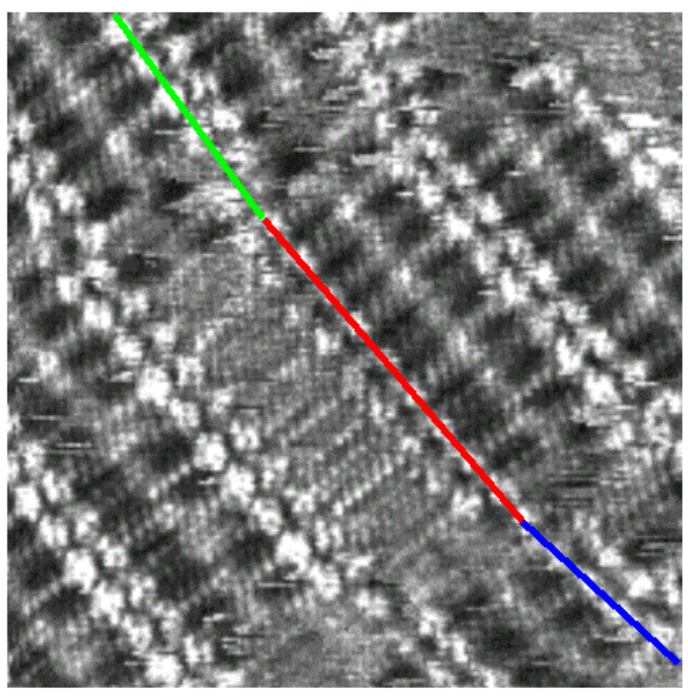

(b)

Figure 4. (a) STM image of a monolayer of F6H11-ISA and H14-ISA adsorbed on graphite. The green arrow indicates a F6H11-ISA lamella. The narrow H14ISA lamella is indicated by the red arrow. A H14-ISA molecule coadsorbed in the F6H11-ISA monolayer is indicated by the yellow arrow. The image size: $8.3 \mathrm{x}$ $8.3 \mathrm{~nm}^{2}$. $\mathrm{I}_{\text {set }}=1.0 \mathrm{nA}$, and $\mathrm{V}_{\text {bias }}=696 \mathrm{mV}$. (b) STM image of a monolayer of F6H11-ISA and H14-ISA adsorbed on graphite. Three domains that make a small angle with each other are visible. The lamellar axis for each of the three domains is indicated by a solid line. In the domain in the center of the image a H14-ISA lamella can be recognized. No domains consisting only of H14-ISA molecules can be detected. The image size is $13.4 \times 13.4 \mathrm{~nm}^{2} . \mathrm{I}_{\text {set }}=1.0 \mathrm{nA}$, and $\mathrm{V}_{\text {bias }}=796 \mathrm{mV}$. 

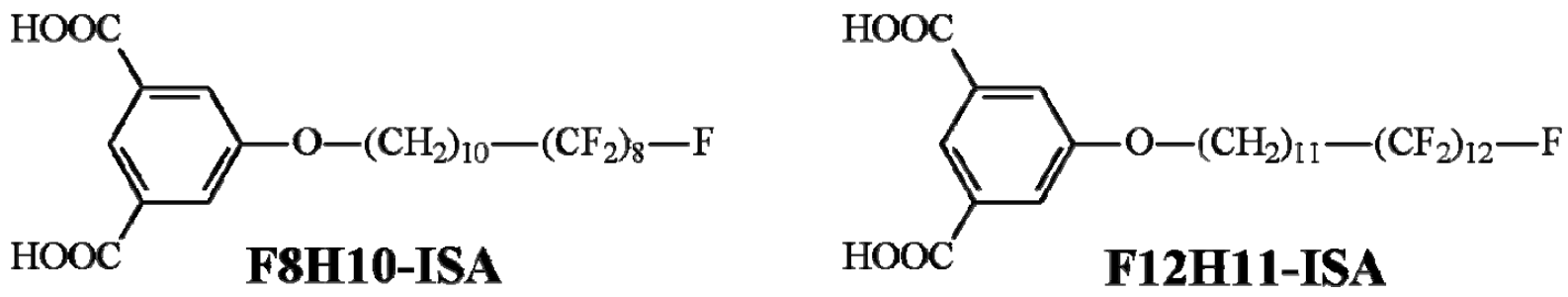

Figure 5. Chemical structure of F8H10-ISA and F12H11-ISA

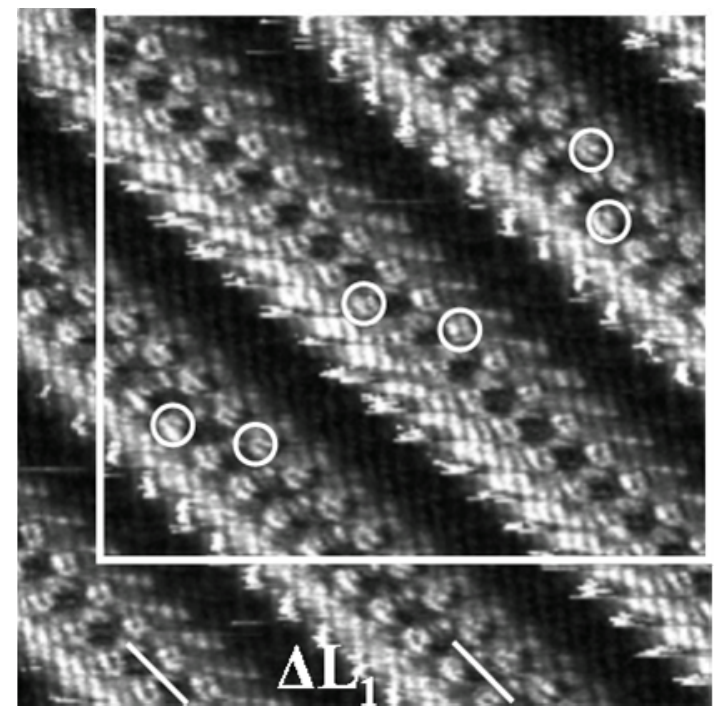

(a)

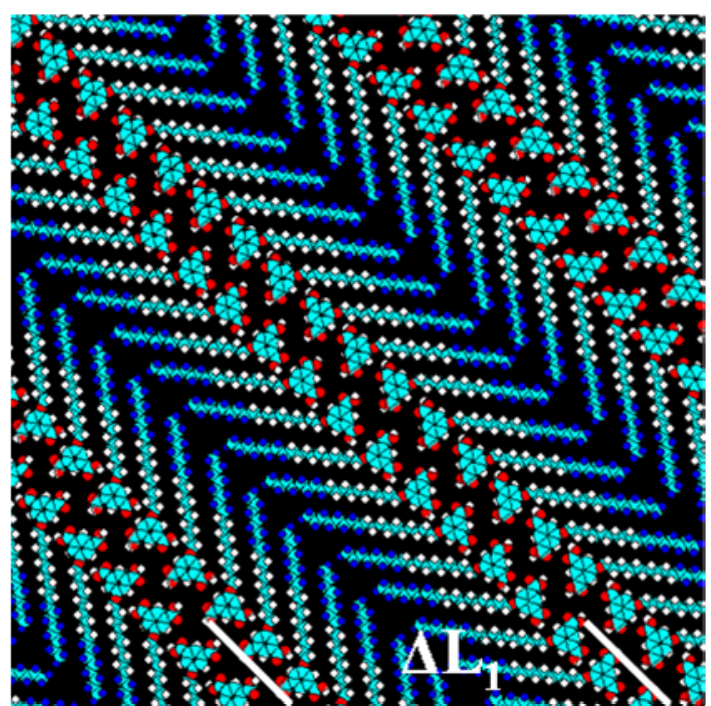

(b)

Figure 6. (a) STM image of F8H10-ISA monolayer deposited from 1-octanol solution on to the basal plane of graphite. The image is submolecularly resolved. Alternating dark and bright rows can be clearly distinguished. The dark row corresponds to the fluorinated part of the molecule, while the bright row corresponds to the non-fluorinated part plus the isophthalic acid group (indicated by white circles). $\Delta \mathrm{L}_{1}$ indicates a lamella. Image size is $10.6 \times 10.6 \mathrm{~nm}^{2} . I_{\text {set }}=$ $1.0 \mathrm{nA}, V_{\text {bias }}=-0.72 \mathrm{~V}$. (b) molecular model for the area indicated in $\mathrm{a}$. 


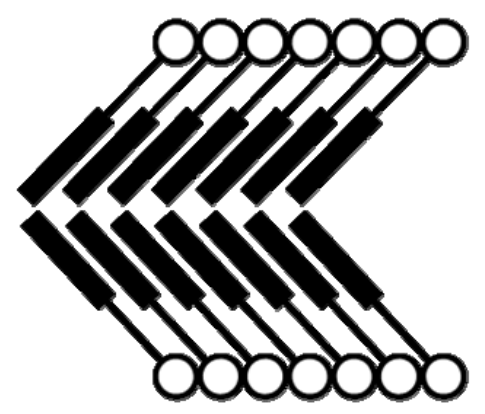

(a)

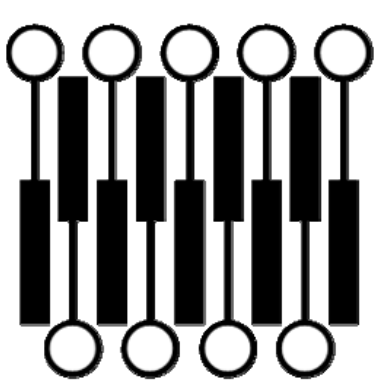

(b)

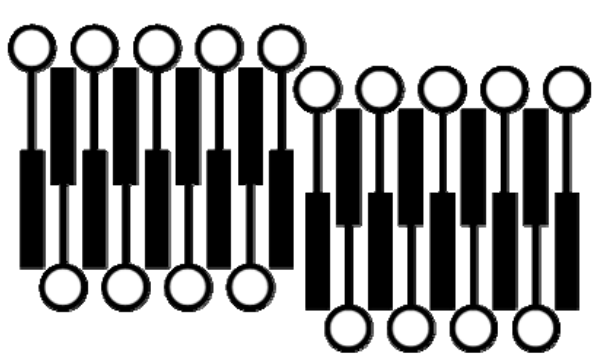

(c)

Scheme 1: Overview of the different types of patterns observed and their relation to phase-separation on the nanoscale: the molecules are depicted as follows: isophthalic acid groups (open circles); non-fluorinated segments (line); perfluorinated segments (bar). (a) nano-phase separation: the isophthalic acid groups, the non-fluorinated segments and the perfluorinated segments form separate zones. (b) No nano-phase separation: interdigitation of the semifluorinated alkyl chains. (c) No nano-phase separation: interdigitation of the semifluorinated alkyl chains in addition to the formation of clusters, indicating the stress enforced by the incompatibility of the non-fluorinated and perfluorinated segments.

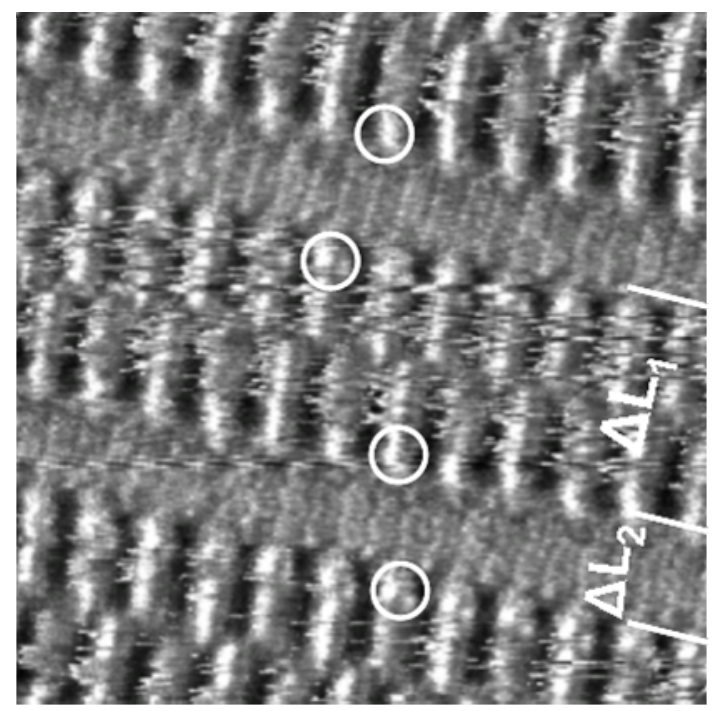

(a)

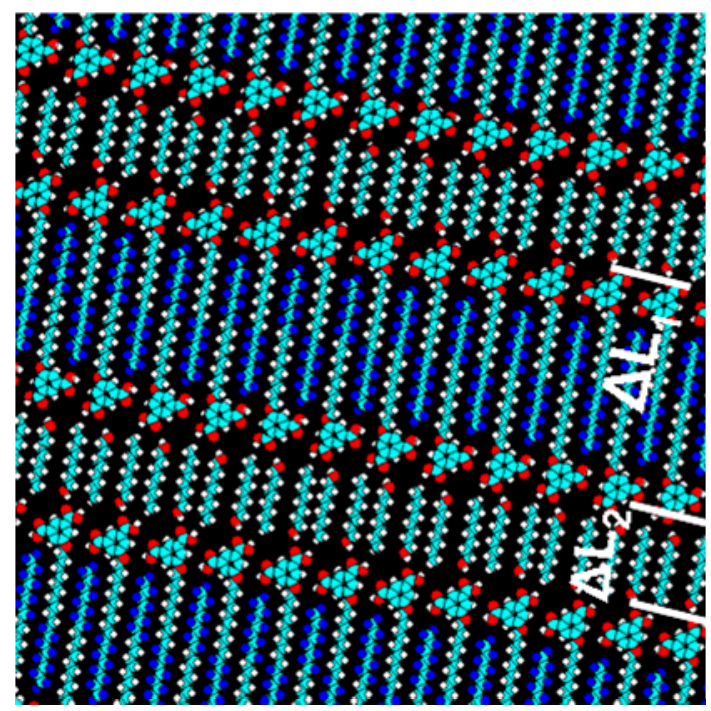

(b)

Figure 7. (a) STM image of a monolayer of F8H10-ISA with no nano-phase separation observed. $\Delta \mathrm{L} 1$ indicates a F8H10-ISA lamella while $\Delta \mathrm{L} 2$ indicates a solvent lamella. Image size $10.1 \times 10.1 \mathrm{~nm} 2$. Iset $=1.0 \mathrm{nA}$, Vbias $=-0.67 \mathrm{~V}$. (b) molecular model of the STM image shown in a. 


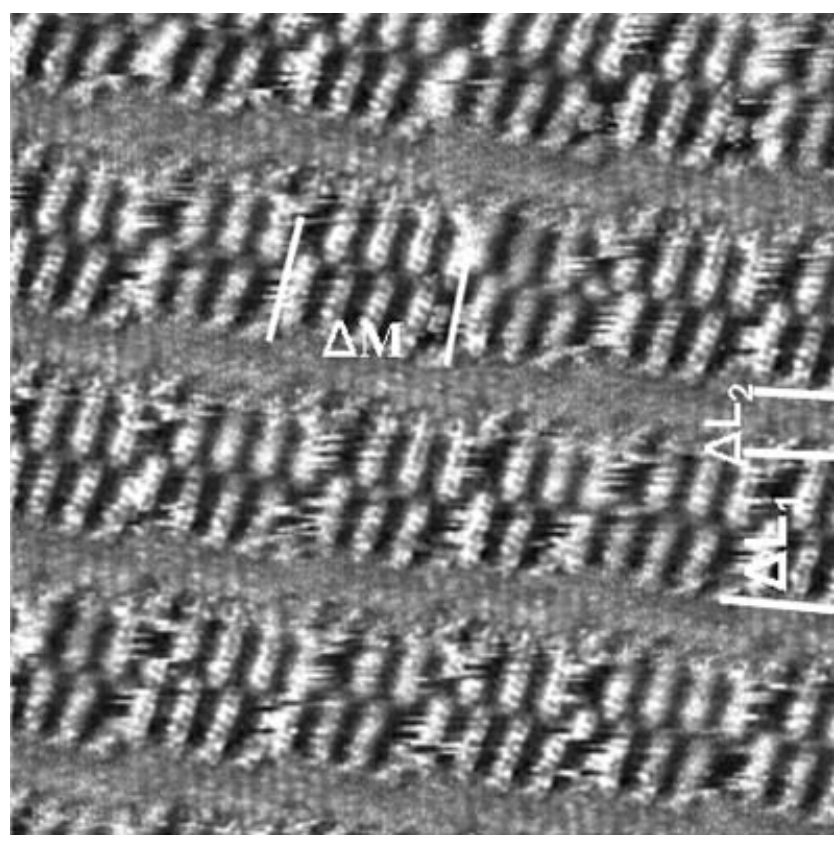

(a)

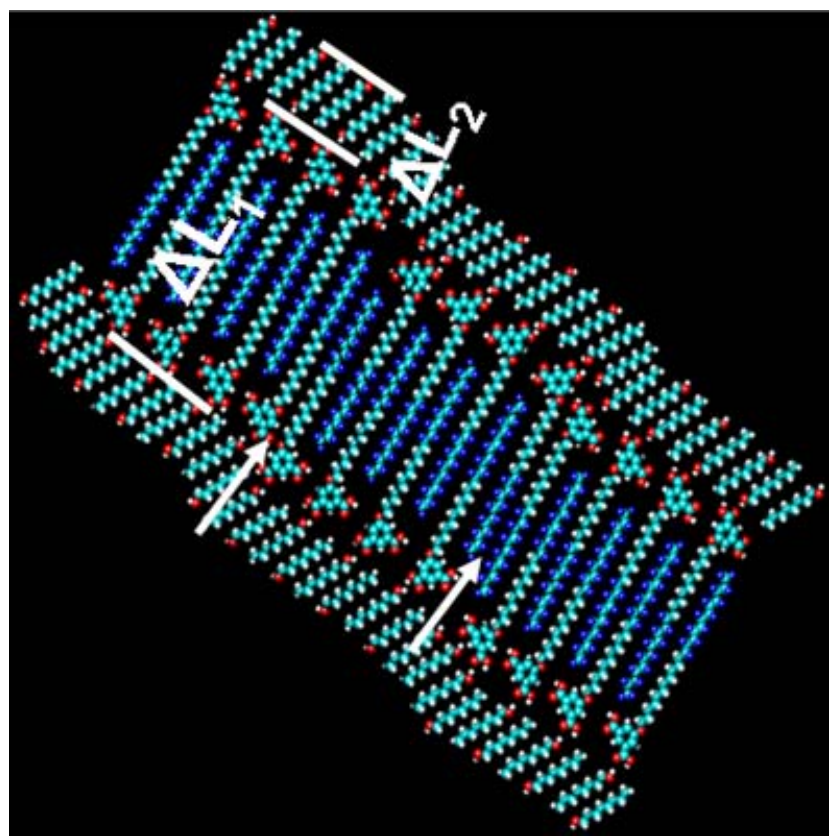

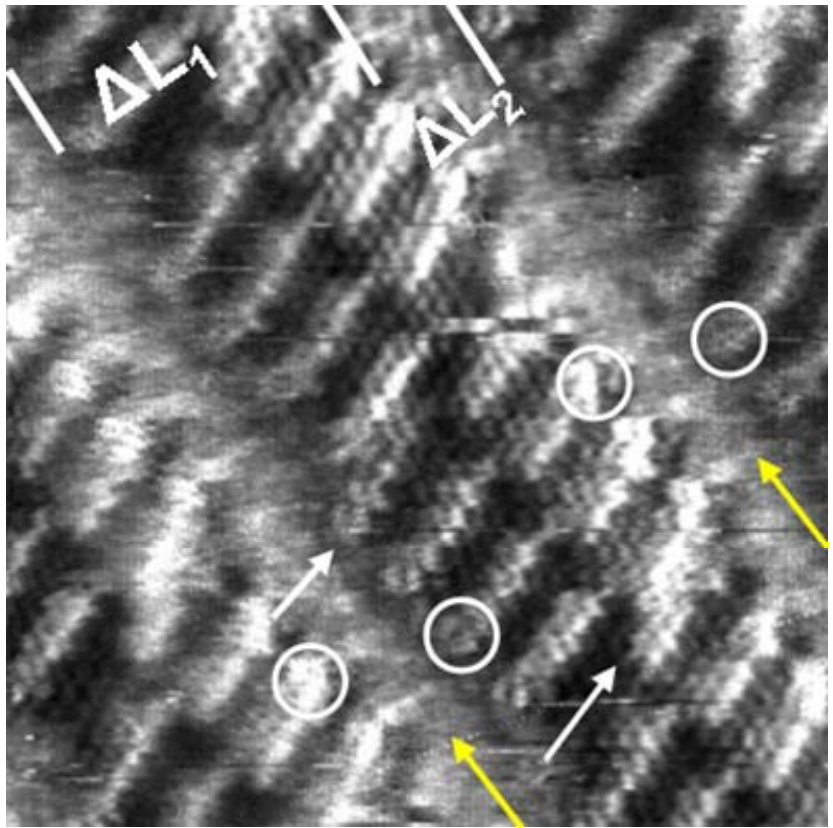

(b)

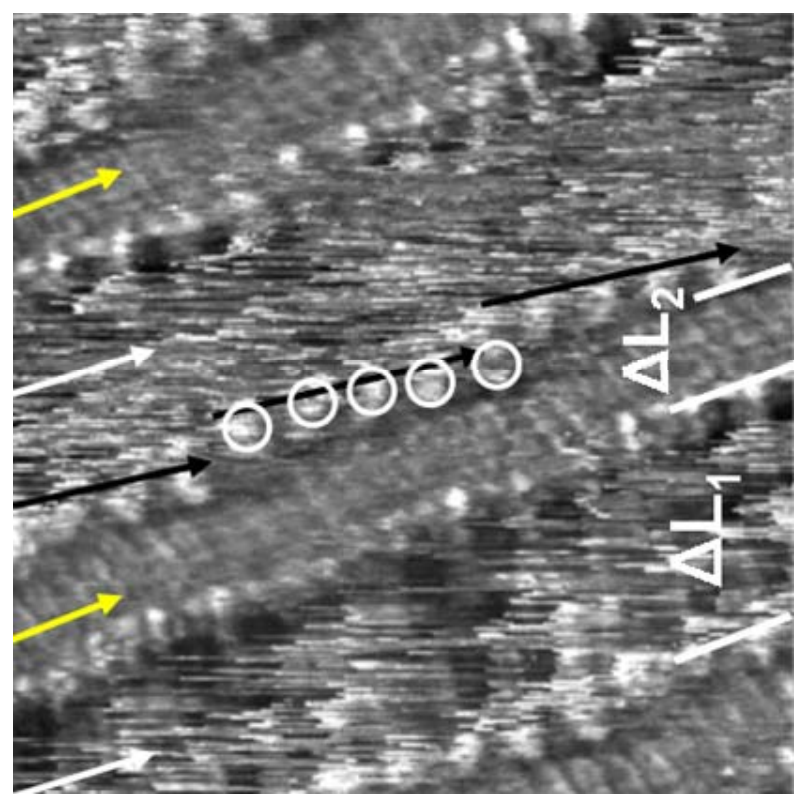

Figure 8. (a) Large scale STM image of the monolayer of F12H11-ISA molecules physisorbed at the 1-octanol - graphite interface. The grouping of the molecules into units of 8 molecules is evident. One unit is indicated by $\Delta \mathrm{M} . \Delta \mathrm{L} 1$ indicates an $\mathrm{F} 12 \mathrm{H} 11-\mathrm{ISA}$ lamella, while $\Delta \mathrm{L} 2$ indicates a solvent lamella. Image size is $20.8 \times 20.8 \mathrm{~nm} 2$. Iset $=0.9 \mathrm{nA}$, and Vset $=-0.7 \mathrm{~V}$. (b) Small scale STM image of a monolayer formed by physisorption of F12H11-ISA molecules at the 1-octanol graphite interface. White arrows point at the interface between two units. White circles indicate the location of some isophthalic acid groups. $\Delta \mathrm{L} 1$ indicates a $\mathrm{F} 12 \mathrm{H} 11-\mathrm{ISA}$ lamella, while $\Delta \mathrm{L} 2$ and the yellow arrows indicate solvent lamellae. Image size is $10.4 \times 10.4 \mathrm{~nm} 2$. Iset $=0.8 \mathrm{nA}$, and Vset $=1.0 \mathrm{~V}$. (c) Molecular model of the F12H11-ISA lamella flanked by two solvent lamellae, indicated by the two yellow arrows in b. (d) STM image of a monolayer of F12H11-ISA. Codeposited solvent lamellae $(\Delta \mathrm{L} 2)$ are indicated by yellow arrows. White circles indicate the location of isophthalic acid groups. The black arrows indicate different units. White arrows point to the area where the $\mathrm{F} 12 \mathrm{H} 11$ part of the molecules is located. 

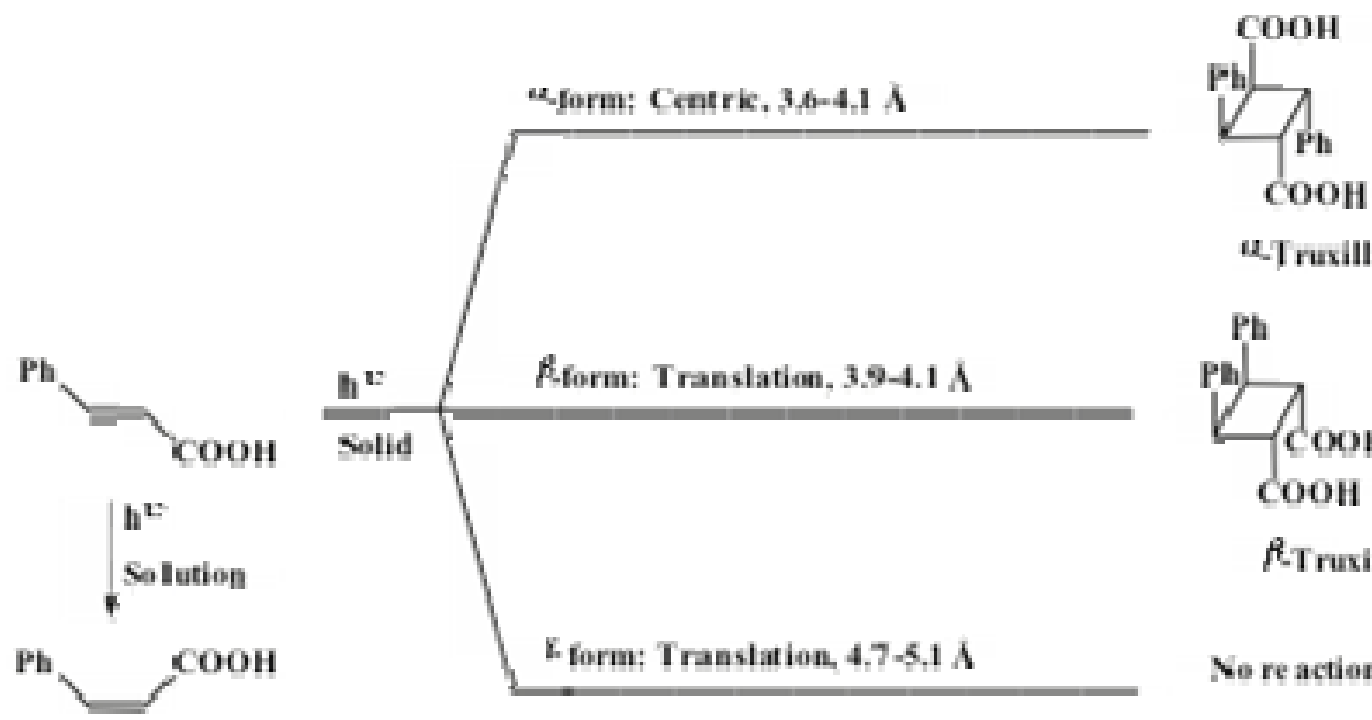

uT ruxillik acid

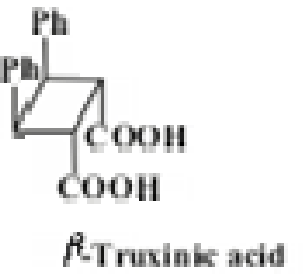

Ne re action

Scheme 2. The reaction products of the different packing patterns of cinnamic acid crystals upon irradiation. The different crystal polymorphs, along with the symmetry relation and distance between reactive molecules are indicated.

CH: eats 0

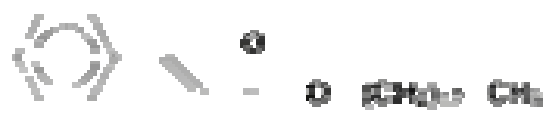
ex

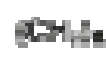<smiles>O=[Sb]1C=C2C=CC=C1C2</smiles>

a Eteimcis Clasmertit

Figure . Chemical structure of $\mathrm{C} 18 \mathrm{cinnC} 18$ and $\mathrm{C} 18 \mathrm{CinnC} 10$ 

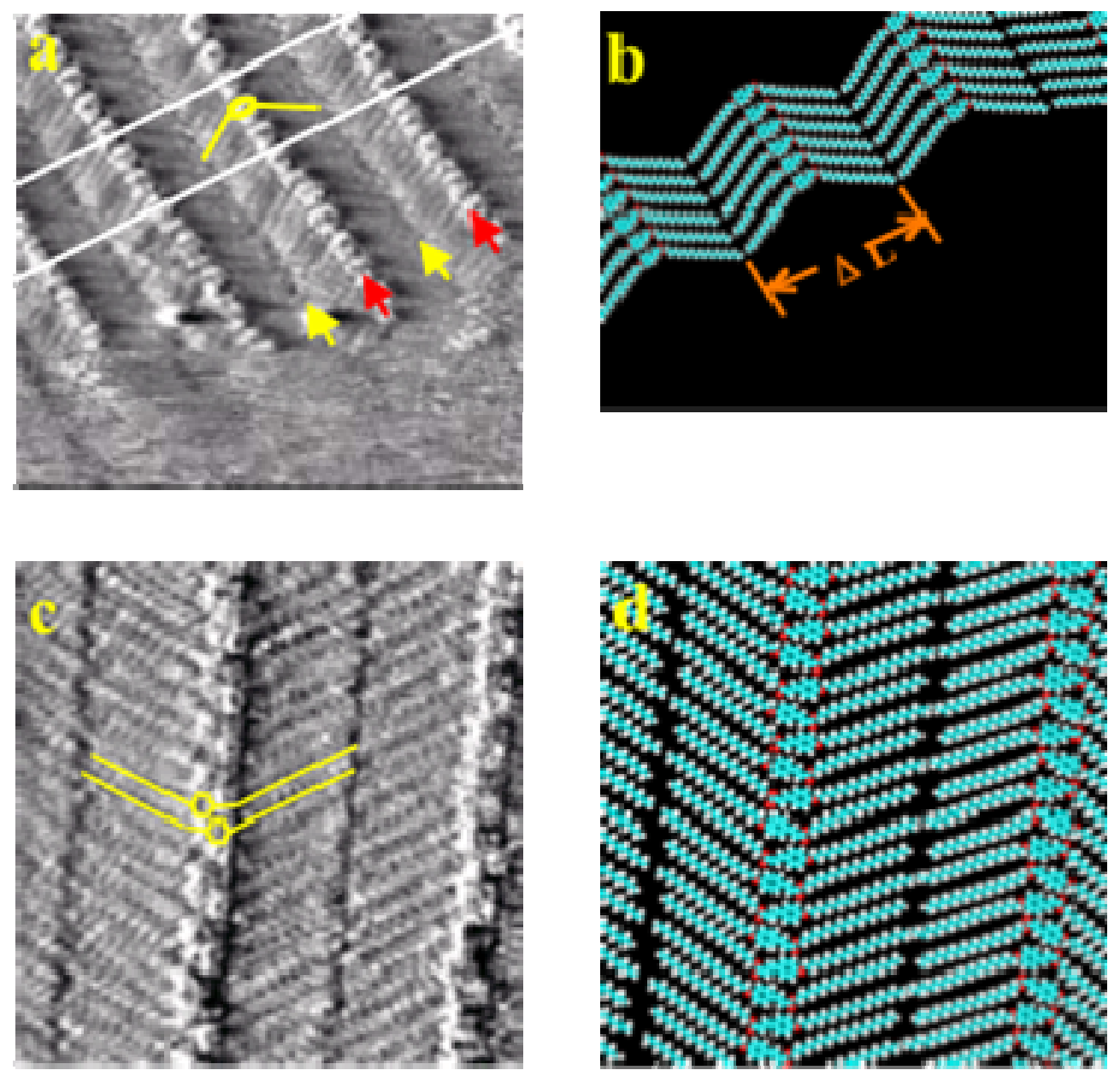

Figure 9. (a) STM image showing a Type 1 monolayer of C18CinnC18 physisorbed at the graphite/liquid interface. Red arrows point to the cinnamate groups, yellow arrows define the lamella boundaries. One molecule is schematically drawn. The image area is $16 \times 16 \mathrm{~nm}^{2}, V_{\text {set }}=-0.312 \mathrm{~V}, \mathrm{I}_{\text {set }}=1.0 \mathrm{nA}$. (b) Molecular model of the outlined area in a where $\Delta \mathrm{L}=54 \pm 3 \AA$, and $a=6.3 \pm 0.5 \AA$. (c) STM image showing a Type 2 monolayer of $\mathbf{C} 18$ CinnC18. Two molecules are schematically drawn. The aryl moieties are clearly discerned, they are ordered in a zigzag orientation. The image area is $12 \times 12 \mathrm{~nm}^{2}, \mathrm{~V}_{\text {set }}=-0.48 \mathrm{~V}$, $\mathrm{I}_{\text {set }}=0.7 \mathrm{nA}(\mathrm{d})$ Molecular model of the monolayer packing shown in $\mathrm{c}$. 

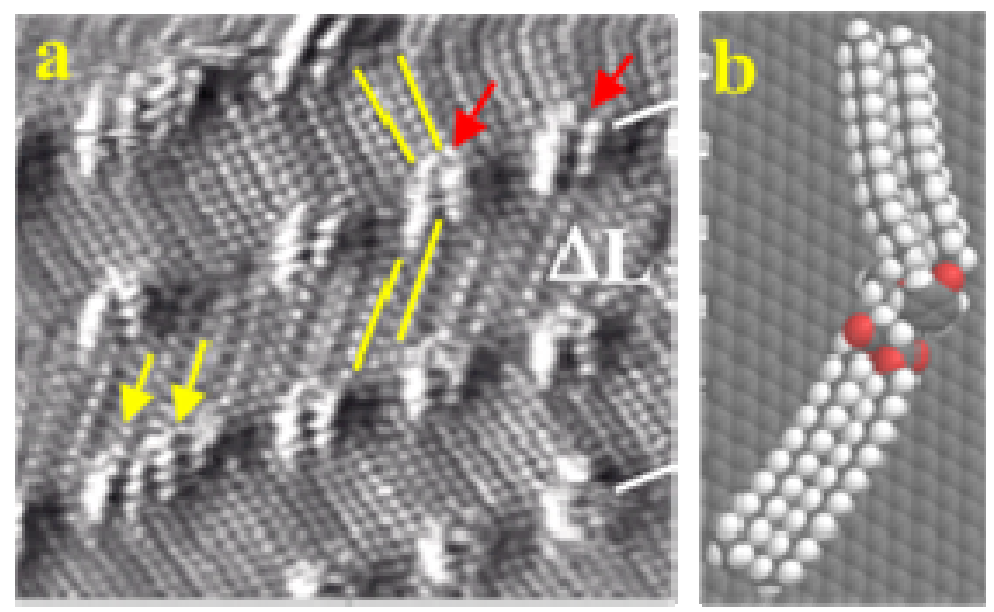

Figure 10. (a) STM image of the dimer. The U-shaped bright structures are correlated with the core of the dimer molecule (red arrows). To each bright structure four alkyl chains are connected (yellow lines). Image area is $10.7 \times 10.7 \mathrm{~nm}^{2}, \mathrm{~V}_{\text {set }}=-0.42 \mathrm{~V}, \mathrm{I}_{\text {set }}$ $=0.7 \mathrm{nA}$ (b) Molecular model showing a dimer molecule on the surface of graphite, after geometry optimization by molecular mechanics calculations.
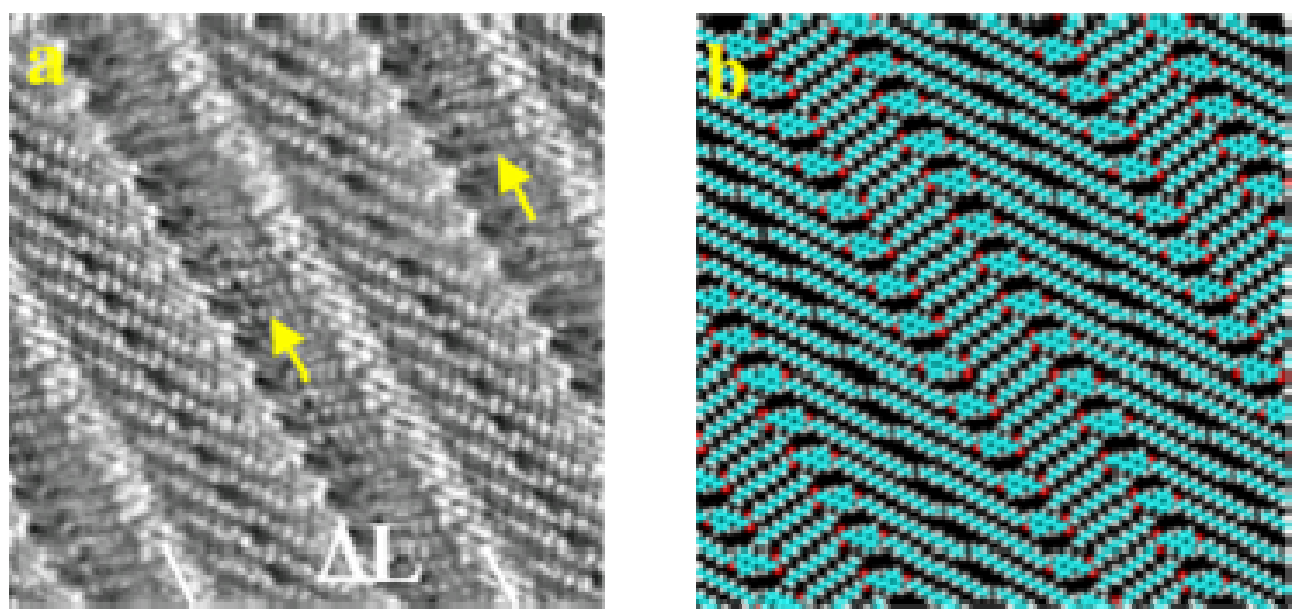

Figure 11. (a) STM image of C18CinnC10. Yellow arrows point to the C10 alkyl chains. The C10 alkyl chains are interdigitating. Image area is $11.3 \times 11.3 \mathrm{~nm}^{2}$, $V_{\text {set }}=-0.304 \mathrm{~V}, \mathrm{I}_{\text {set }}=1.0 \mathrm{nA}(\mathrm{b})$ Molecular model of the monolayer shown in (a). 

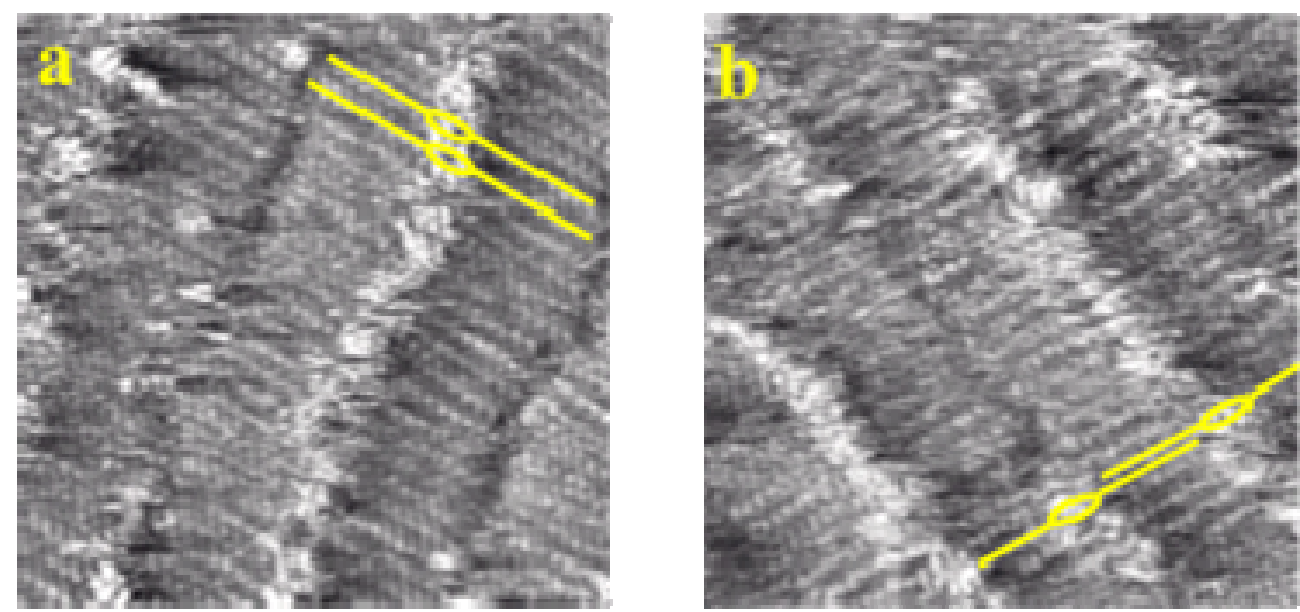

Figure 12. (a) STM image showing the extended conformation of C18CinnC18 monomers. This kind of image is observed after short irradiation intervals in-situ. Two molecules are drawn schematically. Image area is $10.5 \times 10.5 \mathrm{~nm}^{2}, \mathrm{~V}_{\text {set }}=-0.73$ $\mathrm{V}, \mathrm{I}_{\text {set }}=0.75 \mathrm{nA}$ (b) STM image showing the extended conformation of C18CinnC18 monomers. The image shows a part of the monolayer interdigitating (bottom right). Two molecules are drawn schematically. The image area is $10.1 \times 10.1 \mathrm{~nm}^{2}, \mathrm{~V}_{\text {set }}=-$ $0.77 \mathrm{~V}, \mathrm{I}_{\text {set }}=0.6 \mathrm{nA}$.

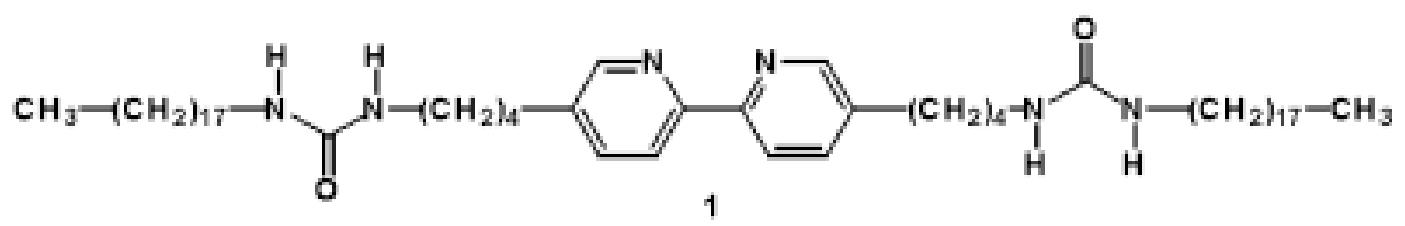

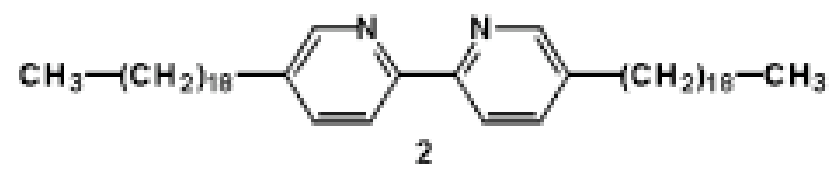

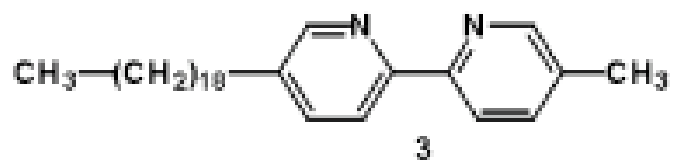

3

Scheme 3. Chemical structures of bipyridine derivatives. 

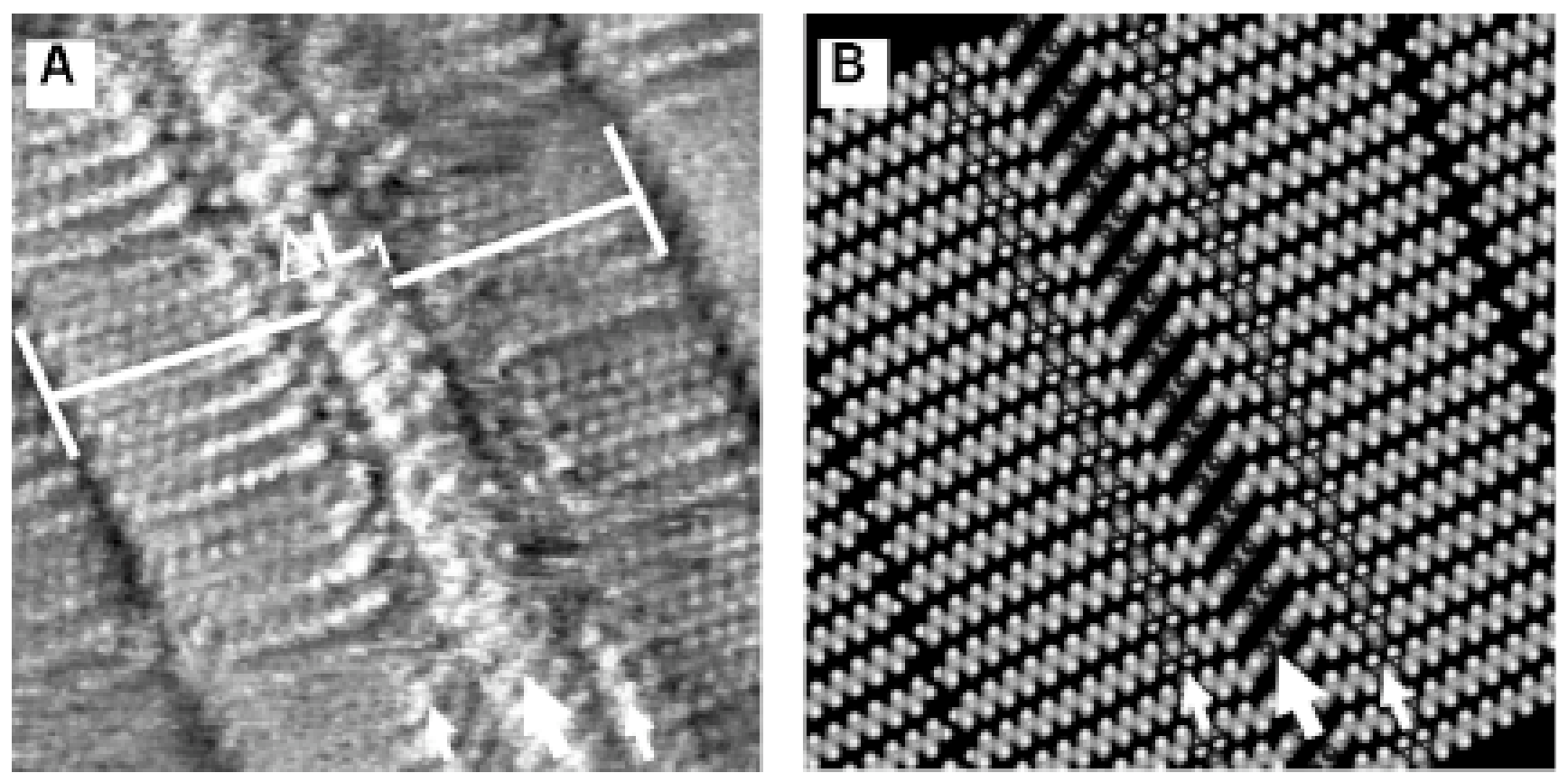

Figure 13. A) STM image of a monolayer composed of molecules of 1 at the liquid/solid interface. Bipyridine moieties appear as a bright tape in the middle of the lamella (big arrow), while the urea groups appear as two bright lines on either side of the bipyridine moieties (small arrows). $\Delta \mathrm{L} 1$ indicates a lamella. The image size is $10.8 \times 10.8 \mathrm{~nm} 2$; Iset $=0.4 \mathrm{nA}$, Vset $=0.304 \mathrm{~V}$. B) A packing model for the monolayer observed in A). The bipyridine moieties are assumed to be tilted off the surface. 

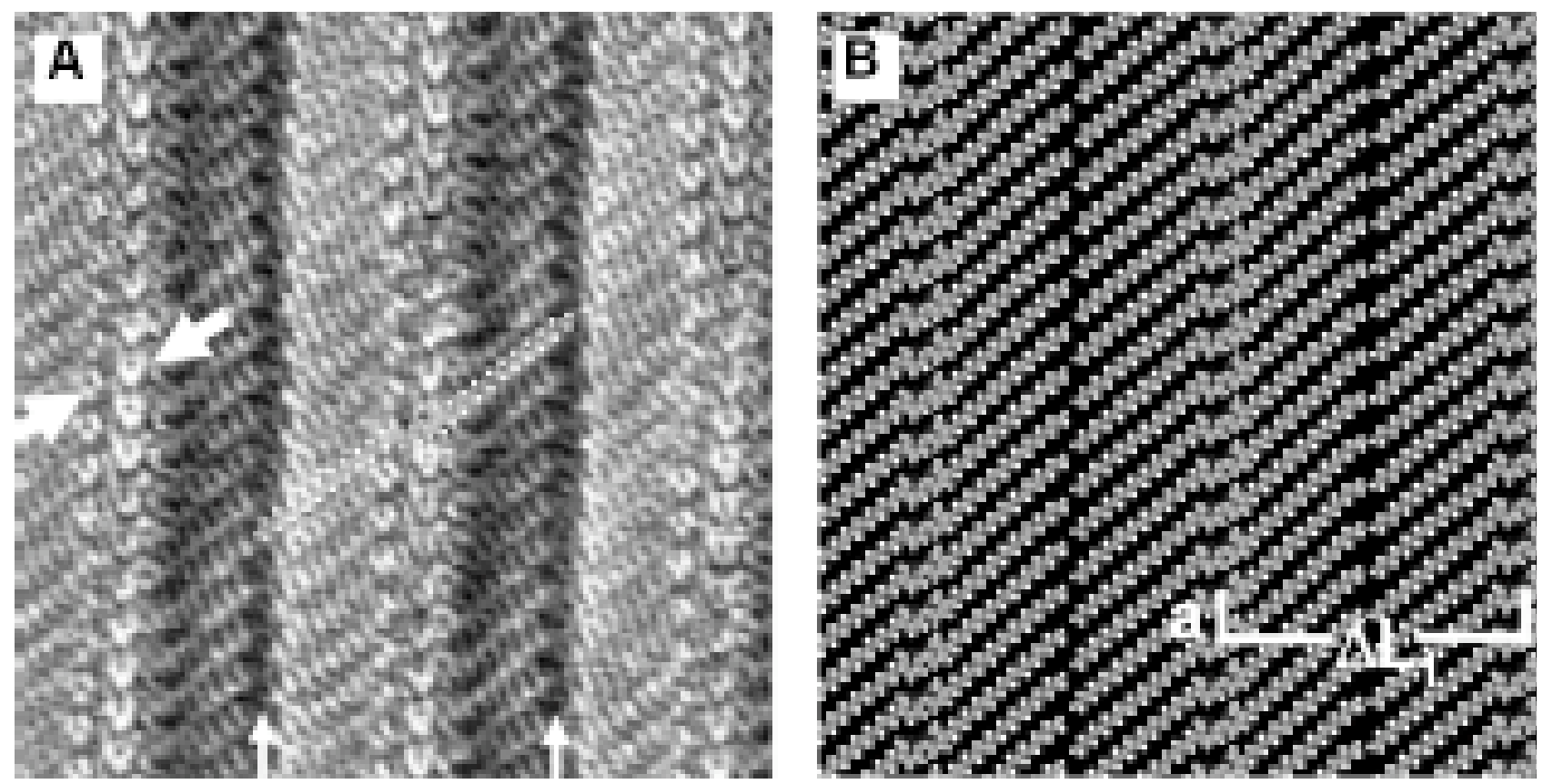

Figure 14. A) STM image of a monolayer of 2 physisorbed at the liquid/solid interface. Big arrows point to the aromatic rings of a bipyridine moiety; small arrows define the lamella boundaries. One molecular model is uperimposed for clarity. The difference in contrast of the alkyl chains on either side of the bipyridine cores is due to a scanning artefact. The image area is $9.1 \times 9.1 \mathrm{~nm} 2$; Iset $=1.2 \mathrm{nA}$, Vset $=0.244 \mathrm{~V}$. B) Molecular model of the monolayer, with $a=6.9 \pm 0.3 \AA, \Delta \mathrm{L}_{1}=51.0 \pm 1.6 \AA$. $A$ cis conformation of the bipyridine units has been assumed arbitrarily. 

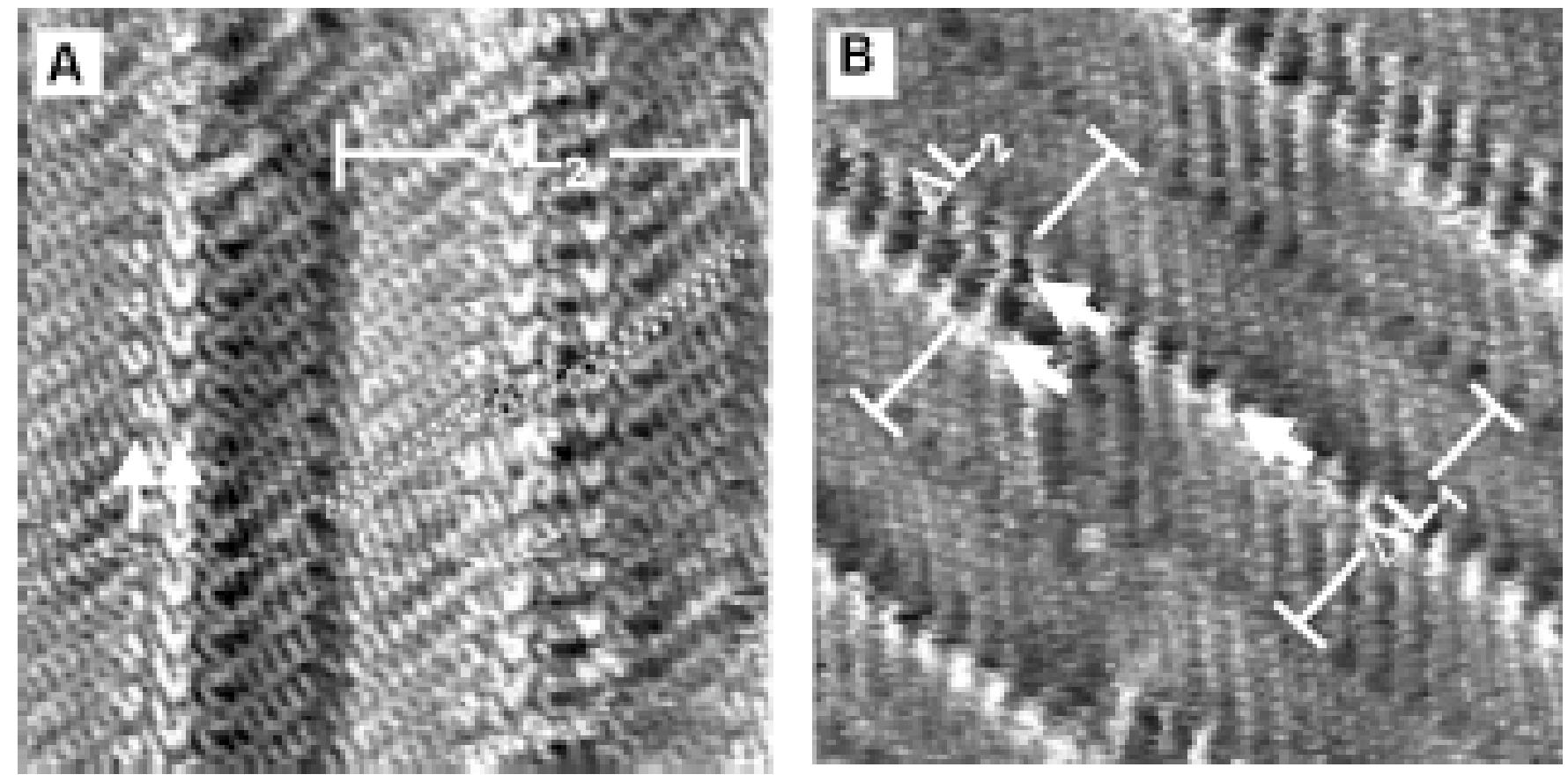

Figure 15. A) STM image of 2 showing two lamellae of different widths. Arrows indicate the rings of the bipyridine units. Two molecular structures are superimposed on the STM image for clarity. $\Delta \mathrm{L} 2$ indicates the width of the wider lamella. The image size is $9 \times 9 \mathrm{~nm} 2$; Iset $=1.2 \mathrm{nA}$, Vset $=-0.242 \mathrm{~V}$. B) STM image of 2 showing a change in the packing pattern within the same lamella. Single and double bipyridine moieties stacked along the lamella direction are indicated by big arrows. The image size is $10.6 \times 10.6 \mathrm{~nm} 2$; Iset $=1.2 \mathrm{nA}$, Vset $=-0.476 \mathrm{~V}$ 


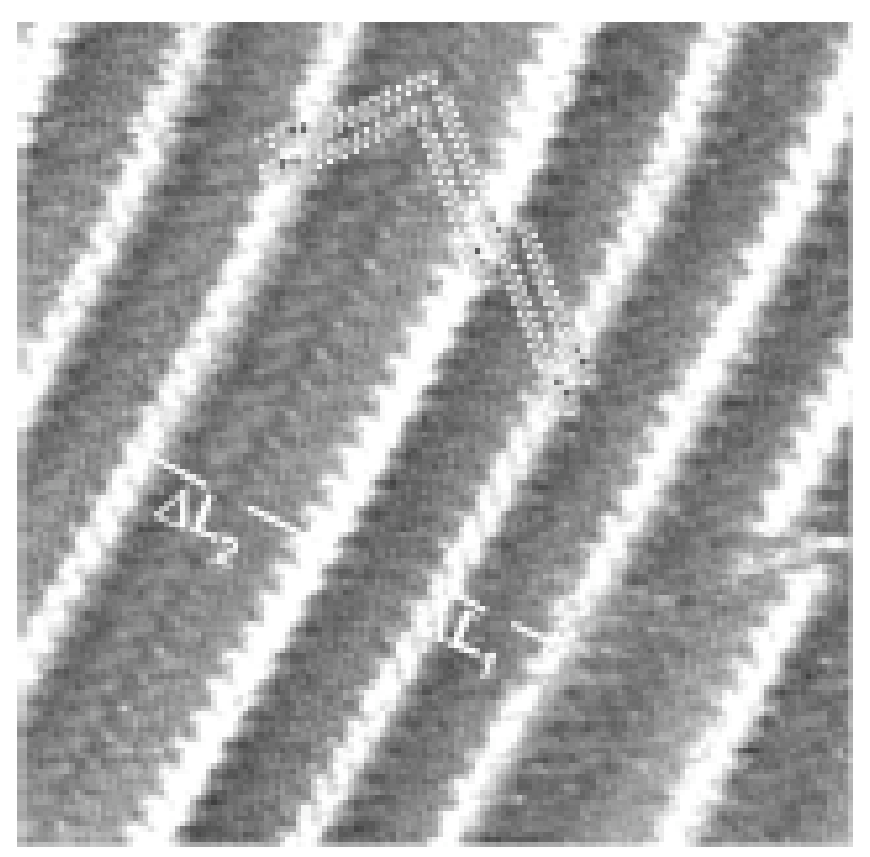

Figure 16. STM image of a monolayer of 3 at the liquid/solid interface. A lamellar structure is evident. The molecules are adsorbed in two different packing patterns, head-to-tail $\left(\Delta \mathrm{L}_{1}=25.5 \pm 1.2 \AA\right)$ and tail-to-tail $\left(\Delta \mathrm{L}_{2}=35.7 \pm 0.9\right.$ $\AA$ ). Six molecular structures are superimposed on the STM image. The image area is $15.2 \times 15.2 \mathrm{~nm}^{2} ; \mathrm{I}_{\text {set }}=0.8 \mathrm{nA}, \mathrm{V}_{\text {set }}=-0.436 \mathrm{~V}$. 

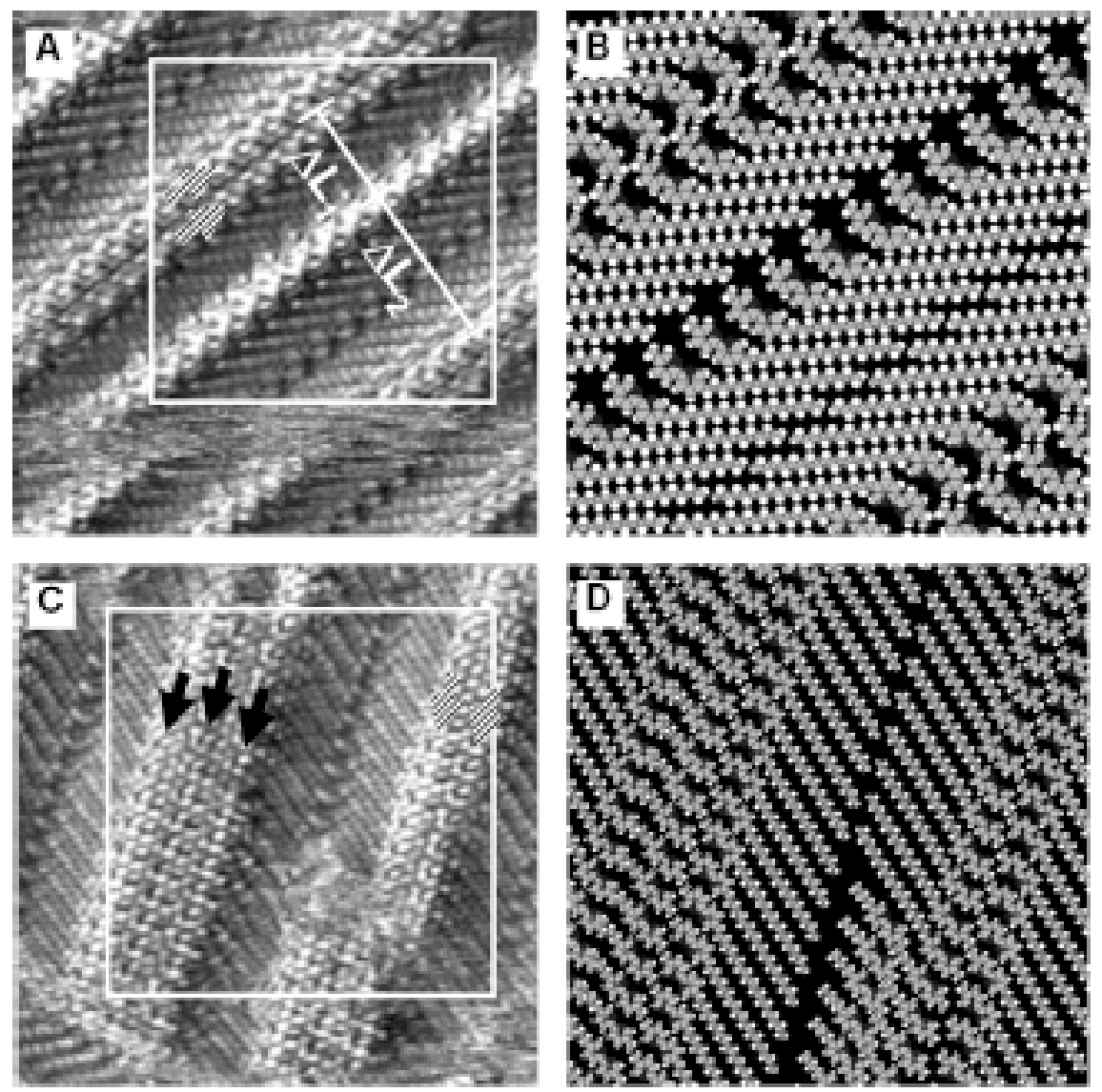

Figure 17. A) STM image of a monolayer of 3 at the liquid/solid interface. Head-totail $(\Delta \mathrm{L} 1)$ and tail-to-tail $(\Delta \mathrm{L} 2)$ packings are observed. Single-core and double-core lamellae are observed (patterned arrows). The image area is $11.5 \times 11.5 \mathrm{~nm} 2$; Iset = $0.8 \mathrm{nA}$, Vset $=-0.4 \mathrm{~V}$. B) Packing model of the area indicated in A). C) STM image of a monolayer of 3 at the liquid/solid interface. A double-core lamella (patterned arrows) and a triple-core lamella (black arrows) can be observed. The image area is $11.5 \times 11.5 \mathrm{~nm} 2$; Iset $=0.8 \mathrm{nA}$, Vset $=-0.5 \mathrm{~V}$. D) Packing model for the area indicated in C). 

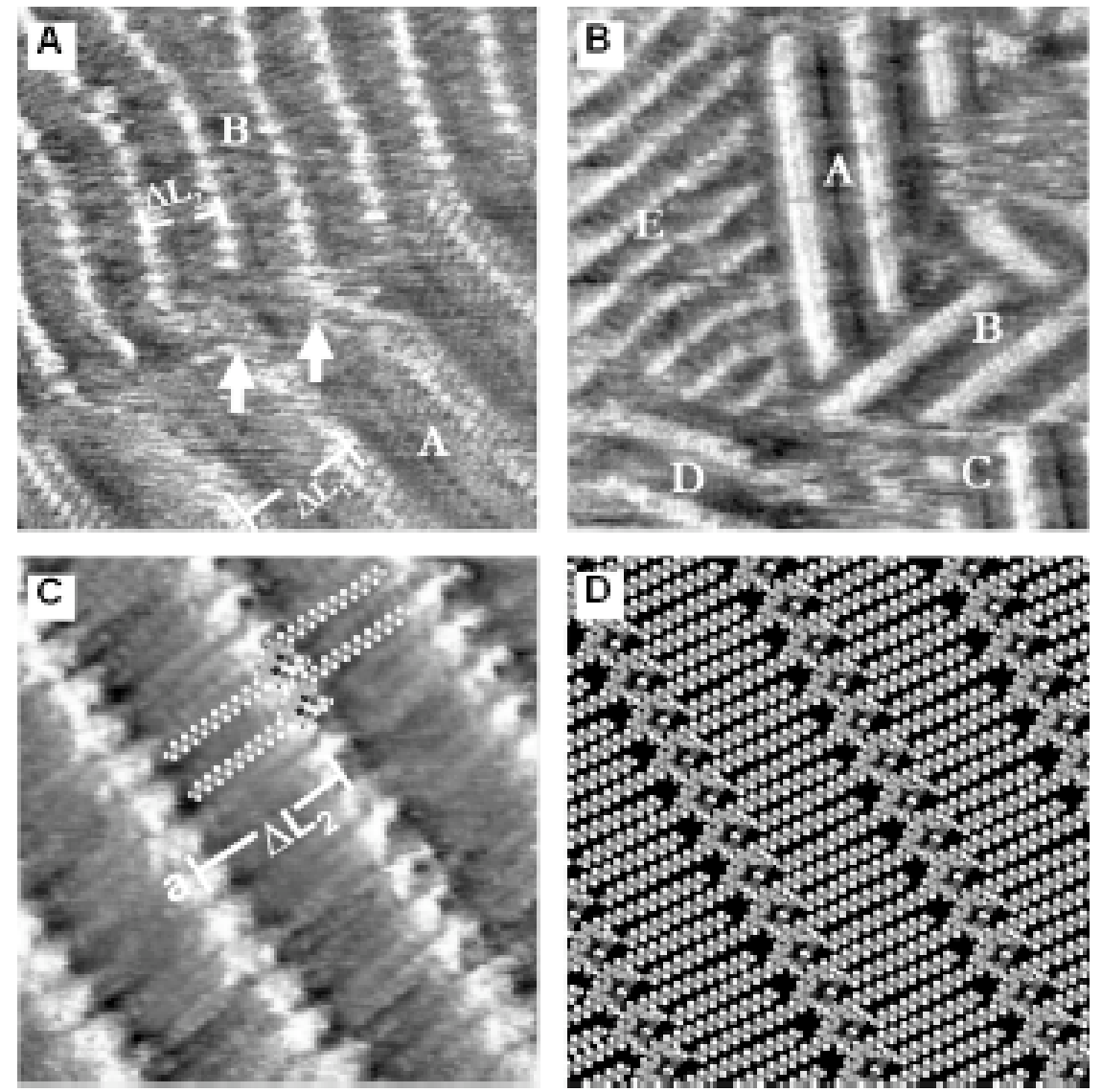

Figure 18. A) STM image showing a monolayer of 2 physisorbed at the liquid/solid interface after addition of $\mathrm{Pd}(\mathrm{OAc}) 2$. Domain $\mathrm{A}$, uncomplexed. Domain $\mathrm{B}$, complexed. $\Delta \mathrm{L} 1=47.5 \pm 1.1 \AA$ and $\Delta \mathrm{L} 2=35.1 \pm 1.3 \AA$. The image size is $21.5 \times 21.5 \mathrm{~nm} 2$; Iset $=0.6$ $\mathrm{nA}$, Vset $=-0.418 \mathrm{~V}$. B) STM image showing uncomplexed domains $A, B, C$, and $D$, while domain $E$ is complexed. The image size is $34.6 \times 34.6 \mathrm{~nm} 2$; Iset $=1.2 \mathrm{nA}$, Vset $=-$ $0.366 \mathrm{~V}$. C) Two complexed molecular structures are superimposed for clarity. The image area is $10.2 \times 10.2 \mathrm{~nm} 2$; Iset $=1.2 \mathrm{nA}$, Vset $=-0.486 \mathrm{~V}$. D) Tentative molecular model of the monolayer where $\mathrm{a}=9.4 \pm 0.1 \AA$ and $\Delta \mathrm{L} 2=35.1 \pm 1.3 \AA$. 

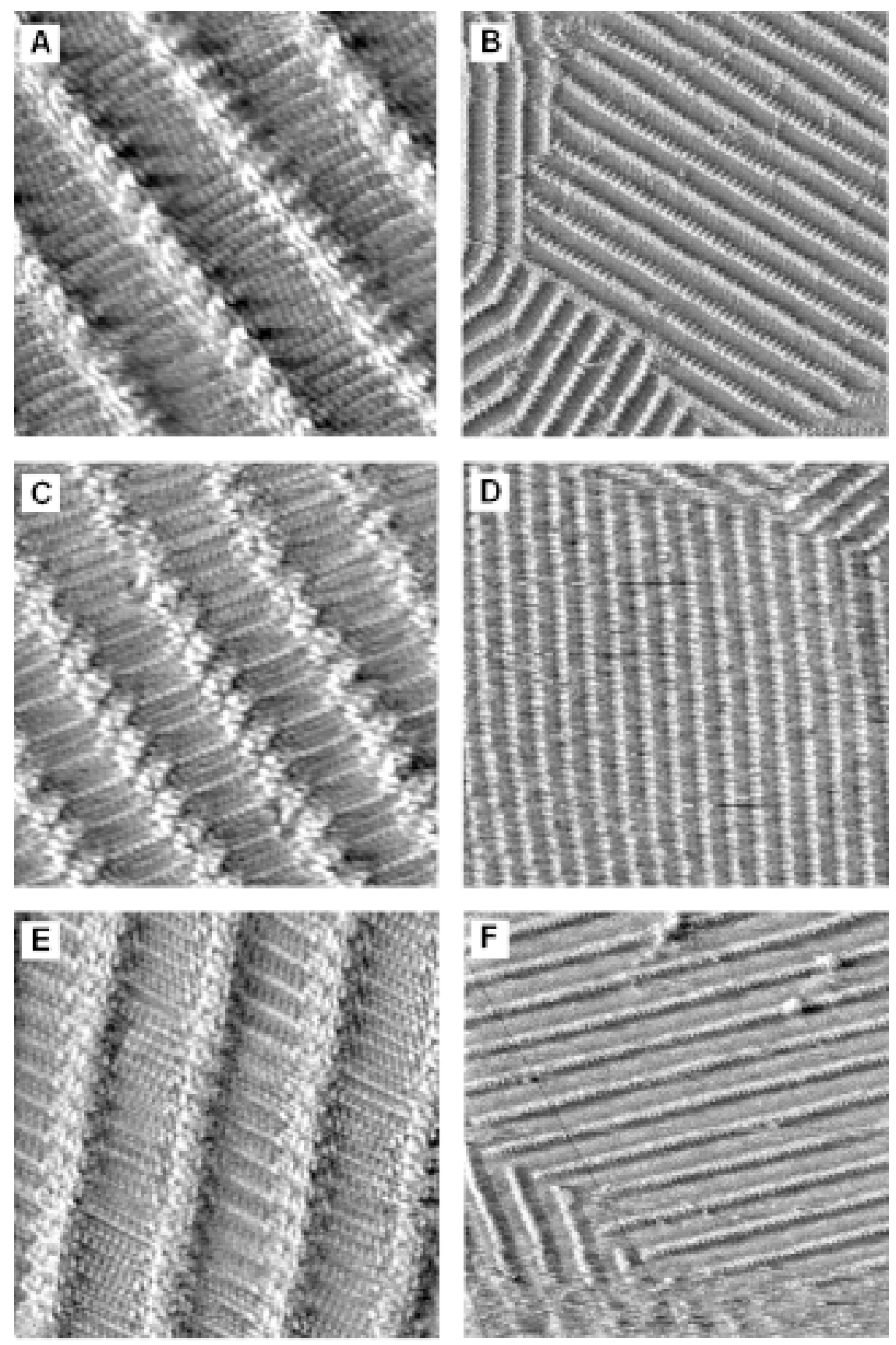

Figure 19. STM images of monolayers of 2 complexed with: (A, B) $\mathrm{PdCl}_{2}: A$ ) The image area is $11.2 \times 11.3 \mathrm{~nm}^{2} ; \mathrm{I}_{\text {set }}=1.2 \mathrm{nA}, \mathrm{V}_{\text {set }}=0.142 \mathrm{~V}$. B) The image area is $50.0 \times 50.0 \mathrm{~nm}^{2} ; \mathrm{I}_{\text {set }}=1.4 \mathrm{nA}, \mathrm{V}_{\text {set }}=0.160 \mathrm{~V}$. (C,D) $\mathrm{Cu}(\mathrm{OAc})_{2}:$ C) The image area is $12.9 \times 12.9 \mathrm{~nm}^{2} ; \mathrm{I}_{\text {set }}=0.8 \mathrm{nA}, \mathrm{V}_{\text {set }}=-0.450 \mathrm{~V}$. D) The image area is $50.0 \times 50.0 \mathrm{~nm}^{2} ; \mathrm{I}_{\text {set }}=0.8 \mathrm{nA}, \mathrm{V}_{\text {set }}=0.472 \mathrm{~V}$. (E, F) $\left.\mathrm{CuCl}_{2}: \mathrm{E}\right)$ The image area is $13.1 \times 13.1 \mathrm{~nm}^{2} ; \mathrm{I}_{\text {set }}=1.0 \mathrm{nA}, V_{\text {set }}=-0.460 \mathrm{~V}$. F) The image area is $50.0 \times 50.0 \mathrm{~nm}^{2}$ $; I_{\text {set }}=0.8 \mathrm{nA}, \mathrm{V}_{\text {set }}=-0.440 \mathrm{~V}$. 

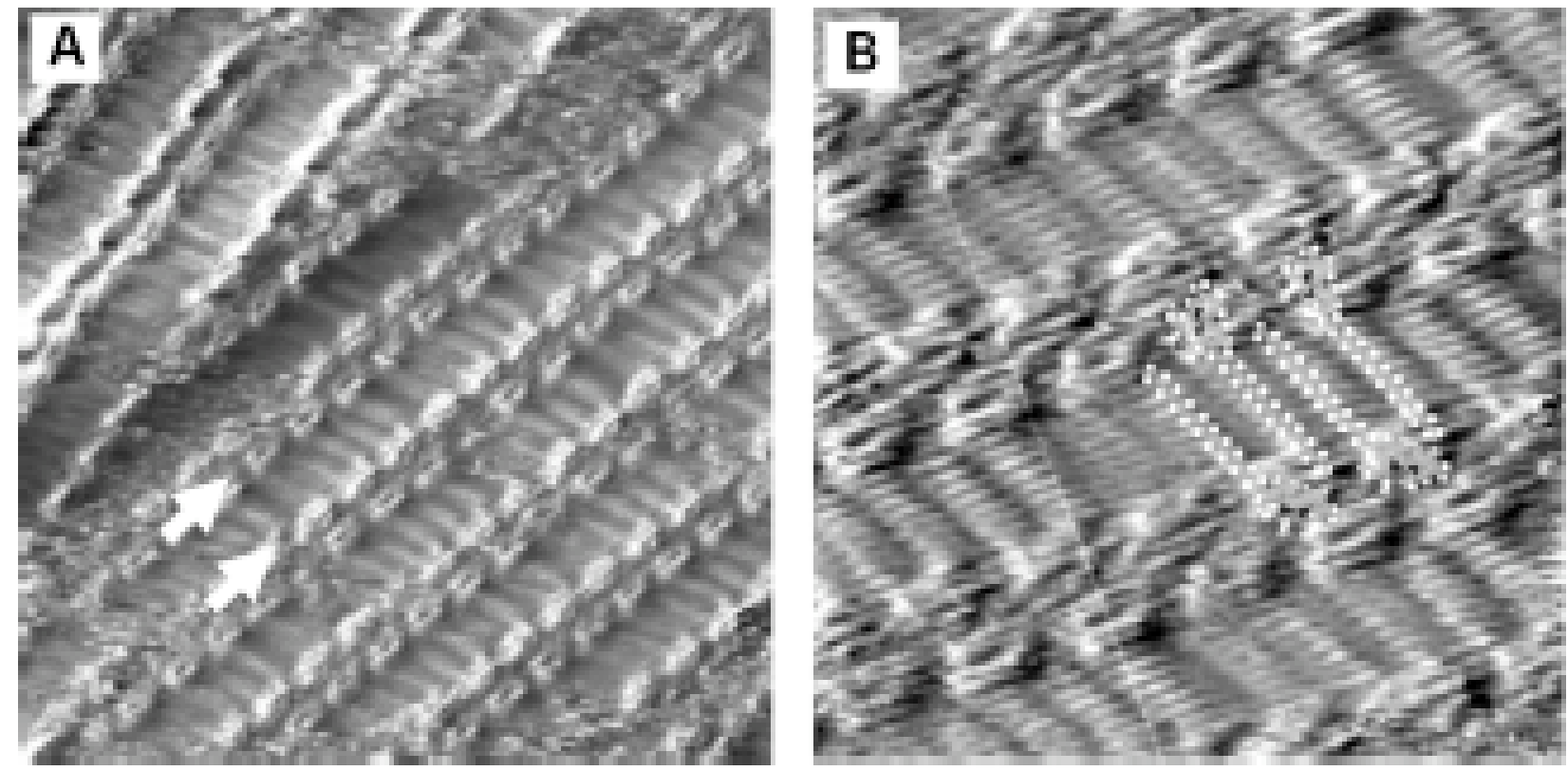

Figure 20. A) Large scale STM image of a monolayer of 3 after the addition of $\mathrm{Pd}(\mathrm{OAc}) 2$. Only one type of packing is observed, in which the alkyl chains are interdigitated. The image area is $24.1 \times 24.1 \mathrm{~nm} 2$; Iset $=1.3 \mathrm{nA}$, Vset $=-0.5 \mathrm{~V}$. B) STM image of a complexed monolayer of 3 . The alkyl chains are clearly interdigitated. The molecular structures of four complexes are superimposed on the image. The image area is $10.8 \times 10.8 \mathrm{~nm} 2$, Iset $=1.2 \mathrm{nA}$, Vset $=-0.6 \mathrm{~V}$. 


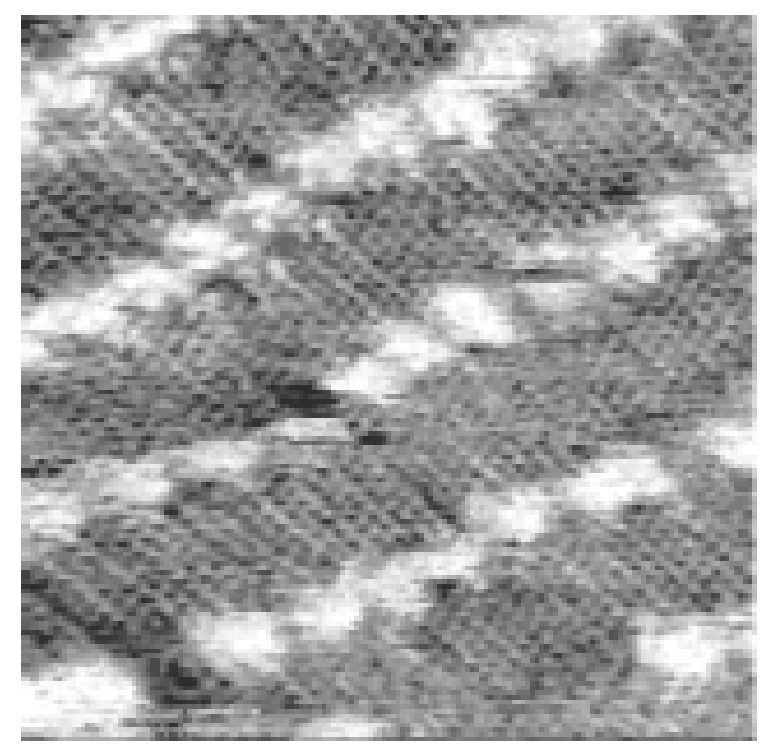

Figure 21. STM image showing a monolayer of 2 physisorbed at the air/solid interface after addition of a solution of $\mathrm{Pd}(\mathrm{OAc})_{2}$ in 1-heptanol and drying for two days under ambient conditions. The image area is $10.2 \times 10.2 \mathrm{~nm}^{2} ; \mathrm{I}_{\text {set }}=1.00 \mathrm{nA}$, $\mathrm{V}_{\text {set }}=-0.498 \mathrm{~V}$. 